\title{
ANALYTIC FAMILIES OF SEMIHYPERBOLIC GENERALIZED POLYNOMIAL-LIKE MAPPINGS
}

\author{
MARIUSZ URBAŃSKI
}

\begin{abstract}
We show that the Hausdorff dimension of Julia sets in any analytic family of semihyperbolic generalized polynomial-like mappings (GPL) depends in a real-analytic manner on the parameter. For the proof we introduce abstract weakly regular analytic families of conformal graph directed Markov systems. We show that the Hausdorff dimension of limit sets in such families is real-analytic, and we associate to each analytic family of semihyperbolic GPLs a weakly regular analytic family of conformal graph directed Markov systems with the Hausdorff dimension of the limit sets equal to the Hausdorff dimension of the Julia sets of the corresponding semihyperbolic GPLs.
\end{abstract}

\section{INTRODUCTION}

The behavior of the pressure function of a semihyperbolic GPL has been studied in [6] and [13] (comp. also [4] and [5]). The approach in [6] was to associate to a given semihyperbolic GPL a Hofbauer tower, whereas in [13] a conformal graph directed Markov system in the sense of [7] was associated. The pressure function was shown to be real-analytic on some interval $(0, u)$ with $u>\operatorname{HD}(J(f))$, and the phase transition phenomenon (break down of real analyticity) was observed for some GPLs in [6]. In the present paper we deal with analytic families of semihyperbolic GPLs and, as the main result, we obtain that the Hausdorff dimension of Julia sets in these families depends in a real-analytic manner on the parameter. To our knowledge this is the first time that the real-analytic dependence of the Hausdorff dimension of Julia sets is established for a family of analytic mappings with critical points in the Julia sets. For hyperbolic maps (in particular, they have no critical points in their Julia sets) this type of result is known in the rational case ([12]) and in the transcendental case (see for e.g. [15], [8], [9] or [3] for a more complete collection of references). Our approach in this paper is to define first weakly regular abstract analytic families of conformal graph directed Markov systems and then to show that the Hausdorff dimension of limit sets in such families varies in a real-analytic way. This is done in Sections 2-4. In Section 5, summarizing the appropriate parts from [13], the construction of associating to each semihyperbolic GPL a conformal graph directed Markov system is described. It is proved in Section 6 that each analytic family of semihyperbolic GPLs gives rise to an analytic family of conformal graph directed Markov systems. Section 7 is devoted to the main step in the proof, namely that the latter family is weakly regular. This simultaneously completes the proof of Theorem 6.6, the main result of this paper.

Date: October 25, 2008.

Key words and phrases. Holomorphic dynamics, Hausdorff dimension, semihyperbolic generalized polynomial-like mappings, real analyticity, Julia sets, conformal graph directed Markov systems.

Research supported in part by the NSF Grant DMS 0400481. 
I would like to add that some assumptions appearing in this paper can certainly be weakened. For instance, the set $U$ in the definition of analytic families of semihyperbolic GPLs may depend in a continuous way on the underlying parameter. Also, one could allow parabolic points. However, for ease of exposition, we restrict the discussion to semi-hyperbolic generalized polynomial-like maps.

ACKNOWLEDGMENT. I would like to thank P. Hassinsky and J. Rivera-letelier for helpful discussions which allowed me to state the results in full generality. I am deeply indebted for an anonymous referee for his/her tedious work to detect all unperfect expressions in the paper.

\section{Conformal Graph Directed Function Systems}

In this section we begin our study of graph directed Markov systems culminating in Section 4 with the proof of real analyticity of the Hausdorff dimension function of limit sets of a weak regularly analytic family of strongly regular conformal graph directed Markov systems. Let us recall the definition of these systems taken from [7]. Graph directed Markov systems are based on a directed multigraph and an associated incidence matrix, $(V, E, i, t, A)$. The multigraph consists of a finite set $V$ of vertices and a countable (either finite or infinite) set of directed edges $E$ and two functions $i, t: E \rightarrow V$. For each edge $e$, $i(e)$ is the initial vertex of the edge $e$ and $t(e)$ is the terminal vertex of $e$. The edge goes from $i(e)$ to $t(e)$. Also, a function $A: E \times E \rightarrow\{0,1\}$ is given, called an incidence matrix. The matrix $A$ is an edge incidence matrix. It determines which edges may follow a given edge. So, the matrix has the property that if $A_{u v}=1$, then $t(u)=i(v)$. We will consider finite and infinite walks through the vertex set consistent with the incidence matrix. Thus, we define the set of infinite admissible words $E_{A}^{\infty}$ on an alphabet $A$,

$$
E_{A}^{\infty}=\left\{\omega \in E^{\infty}: A_{\omega_{i} \omega_{i+1}}=1 \text { for all } i \geq 1\right\}
$$

by $E_{A}^{n}$ we denote the set of all subwords of $E_{A}^{\infty}$ of length $n \geq 1$, and by $E_{A}^{*}$ we denote the set of all finite subwords of $E_{A}^{\infty}$. We will consider the left shift map $\sigma: E_{A}^{\infty} \rightarrow E_{A}^{\infty}$ defined by dropping the first entry of $\omega$. Sometimes we also consider this shift as being defined on words of finite length. Given $\omega \in E^{*}$ by $|\omega|$ we denote the length of the word $\omega$, i.e. the unique $n$ such that $\omega \in E_{A}^{n}$. If $\omega \in E_{A}^{\infty}$ and $n \geq 1$, then

$$
\left.\omega\right|_{n}=\omega_{1} \ldots \omega_{n} \text {. }
$$

A Graph Directed Markov System (GDMS) consists of a directed multigraph $(V, E, i, t, A)$ (with an incidence matrix $A$ ) together with a set of non-empty compact metric spaces $\left\{X_{v}\right\}_{v \in V}$, a real number $s \in(0,1)$, and a collection $\phi_{e}: X_{t(e)} \rightarrow X_{i(e)}, e \in E$, of a 1-to-1 contractions with Lipschitz constants bounded above by $s$. Briefly, the set

$$
\Phi=\left\{\phi_{e}: X_{t(e)} \rightarrow X_{i(e)}\right\}_{e \in E}
$$

is called a GDMS. We now describe its limit set. For each $\omega \in E_{A}^{*}$, say $\omega=\omega_{1} \omega_{2} \ldots \omega_{n} \in$ $E_{A}^{n}$, we consider the map coded by $\omega$,

$$
\phi_{\omega}=\phi_{\omega_{1}} \circ \cdots \circ \phi_{\omega_{n}}: X_{t\left(\omega_{n}\right)} \rightarrow X_{i\left(\omega_{1}\right)} .
$$


For $\omega \in E_{A}^{\infty}$, the sets $\left\{\phi_{\left.\omega\right|_{n}}\left(X_{t\left(\omega_{n}\right)}\right)\right\}_{n \geq 1}$ form a descending sequence of non-empty compact sets and therefore $\bigcap_{n \geq 1} \phi_{\left.\omega\right|_{n}}\left(X_{t\left(\omega_{n}\right)}\right) \neq \emptyset$. Since for every $n \geq 1, \operatorname{diam}\left(\phi_{\left.\omega\right|_{n}}\left(X_{t\left(\omega_{n}\right)}\right)\right) \leq$ $s^{n} \operatorname{diam}\left(X_{t\left(\omega_{n}\right)}\right) \leq s^{n} \max \left\{\operatorname{diam}\left(X_{v}\right): v \in V\right\}$, we conclude that the intersection

$$
\bigcap_{n \geq 1} \phi_{\left.\omega\right|_{n}}\left(X_{t\left(\omega_{n}\right)}\right)
$$

is a singleton and we denote its only element by $\pi(\omega)$. In this way we have defined the coding map $\pi$ :

$$
\pi=\pi_{\Phi}: E_{A}^{\infty} \rightarrow X:=\bigcup_{v \in V} X_{v}
$$

from $E^{\infty}$ to $\bigcup_{v \in V} X_{v}$, the disjoint union of the compact sets $X_{v}$. The set

$$
J=J_{\Phi}=\pi\left(E_{A}^{\infty}\right)
$$

will be called the limit set of the GDMS $\Phi$. We call a GDMS conformal (CGDMS) if the following conditions are satisfied.

(4a) For every vertex $v \in V, X_{v}$ is a compact connected subset of a Euclidean space $\mathbb{R}^{d}$ (the dimension $d$ is the same for all $v \in V$ ) and $X_{v}=\overline{\operatorname{Int}\left(X_{v}\right)}$.

(4b) (Open set condition) $(O S C)$ For all $a, b \in E, a \neq b$,

$$
\phi_{a}\left(\operatorname { I n t } ( X _ { t ( a ) } ) \cap \phi _ { b } \left(\operatorname{Int}\left(X_{t(b)}\right)=\emptyset .\right.\right.
$$

(4c) For every vertex $v \in V$ there exists an open connected set $W_{v} \supset X_{v}$ such that for every $e \in E$ with $t(e)=v$, the map $\phi_{e}$ extends to a $C^{1}$ conformal diffeomorphism of $W_{v}$ into $W_{i(e)}$.

(4d) (Cone property) There exist $\gamma, l>0, \gamma<\pi / 2$, such that for every $x \in X \subset \mathbb{R}^{d}$ there exists an open cone $\operatorname{Con}(x, \gamma, l) \subset \operatorname{Int}(X)$ with vertex $x$, central angle of measure $\gamma$, and altitude $l$.

(4e) There are two constants $L \geq 1$ and $\alpha>0$ such that

$$
\left\|\phi_{e}^{\prime}(y)|-| \phi_{e}^{\prime}(x)\right\| \leq L\left\|\left(\phi_{e}^{\prime}\right)^{-1}\right\|^{-1}\|y-x\|^{\alpha},
$$

for every $e \in E$ and every pair of points $x, y \in X_{t(e)}$, where $\left|\phi_{\omega}^{\prime}(x)\right|$ means the norm of the derivative.

We proved in [7] the following remarkable result.

Proposition 2.1. If $d \geq 2$ and a family $\Phi=\left\{\phi_{e}\right\}_{e \in E}$ satisfies conditions (4a) and (4c), then it also satisfies condition (4e) with $\alpha=1$.

As a rather straightforward consequence of (4e) we proved in [7] the following.

Lemma 2.2. If $\Phi=\left\{\phi_{e}\right\}_{e \in E}$ is a CGDMS, then for all $\omega \in E^{*}$ and all $x, y \in W_{t(\omega)}$, we have

$$
|\log | \phi_{\omega}^{\prime}(y)|-\log | \phi_{\omega}^{\prime}(x)|| \leq \frac{L}{1-s}\|y-x\|^{\alpha} .
$$


As a straightforward consequence of (4e) (see [7]), we get the following.

(4f) (Bounded distortion property). There exists $K \geq 1$ such that for all $\omega \in E^{*}$ and all $x, y \in X_{t(\omega)}$

$$
\left|\phi_{\omega}^{\prime}(y)\right| \leq K\left|\phi_{\omega}^{\prime}(x)\right|
$$

It was proved in [7] that for each $t \geq 0$ the following limit exists (which can be equal to $+\infty)$.

$$
\mathrm{P}(t)=\lim _{n \rightarrow \infty} \frac{1}{n} \log \sum_{\omega \in E_{A}^{n}}\left\|\phi_{\omega}^{\prime}\right\|^{t} .
$$

This number is called the topological pressure at the parameter $t$. In [7] a second useful parameter asscociate with a CGDMS has been introduced. Namely,

$$
\theta(\Phi)=\inf \{t: \mathrm{P}(t)<+\infty\}=\sup \{t: \mathrm{P}(t)=+\infty\}
$$

Let $\mathcal{F}$ in $(E)$ denote the family of all finite subsets of $E$. The incidence matrix $A$ is called finitely irreducible if there exists a finite set $\Gamma \subset E_{A}^{*}$ such that for all $a, b \in E$ there exists $\gamma \in \Gamma$ such that $a \gamma b \in E_{A}^{*}$. The minimal cardinality of all such finite sets $\Gamma$ is called the order of the matrix $\Gamma$. If $A$ is finitely irreducible, then the systesm $\Phi$ is also called irreducible, and its order is, by definition, the order of the incidence matrix $A$. The matrix $A$ and the system $\Phi$ are called finitely primitive if there exists set $\Gamma$ as above consisting of words of the same length. The least cardinality of such sets $\Gamma$ is called the order of the system $\Phi$. The following characterization of $\operatorname{HD}\left(J_{\Phi}\right)$ (denoted also by $h_{E}$ ), the Hausdorff dimension of the limit set $J_{\Phi}$, is a version of Bowen's formula. It is proved as proved in [7] as Theorem 4.2.13.

Theorem 2.3. If the a CGDMS $\Phi$ is finitely irreducible, then

$$
\mathrm{HD}\left(J_{\Phi}\right)=\inf \{t \geq 0: \mathrm{P}(t)<0\}=\sup \left\{h_{F}: F \in \mathcal{F} \text { in }(E)\right\} \geq \theta(\Phi) .
$$

If there exists $t \geq 0$ such that $\mathrm{P}(t)=0$, then $t$ is the only zero of the function $\mathrm{P}(t)$, $t=\mathrm{HD}(J)$, and the system $\Phi$ is called regular.

In fact it was assumed in [7] that the system $\Phi$ is finitely primitive, but the proof can easily be improved to this slightly more general setting. It will be convenient for us to make use of the following definitions.

Definition 2.4. A CGDMS is said to be strongly regular if there exists $t \geq 0$ such that $0<\mathrm{P}(t)<\infty$.

A family $\left\{\phi_{i}\right\}_{i \in F}$ is said to be a cofinite subsystem of a system $\Phi=\left\{\phi_{i}\right\}_{i \in E}$ if $F \subset E$ and the difference $E \backslash F$ is finite. 
Definition 2.5. A CGDMS is said to be cofinitely regular if each of its cofinite subsystems is regular.

The following fact, relating all these three notions, is obvious.

Proposition 2.6. Each cofinitely regular system is strongly regular, and each strongly regular system is regular.

Note that the system $\Phi$ is strongly regular if and only if $\operatorname{HD}\left(J_{\Phi}\right)>\theta(\Phi)$.

\section{Analyticity of Perron-Frobenius Operators}

The Section 2.6 from [7] about analyticity of Perron-Frobenius operators and of topological pressure, unfortunately contains imprecise and incorrect statements. However, these flaws are correctable. Since the results of this Section 2.6 from [7] are fundamental in the current paper, we therefore present them here in a corrected way.

Recall that for $\omega, \tau \in E_{A}^{\infty}$, we define $\omega \wedge \tau \in E_{A}^{\infty} \cup E_{A}^{*}$ to be the longest initial block common to both $\omega$ and $\tau$. We say that a function $f: E_{A}^{\infty} \rightarrow \mathbb{C}$ is Hölder continuous with an exponent $\alpha>0$ if

$$
v_{\alpha}(f):=\sup _{n \geq 1}\left\{v_{\alpha, n}(f)\right\}<\infty
$$

where

$$
v_{\alpha, n}(f)=\sup \left\{|f(\omega)-f(\tau)| e^{\alpha(n-1)}: \omega, \tau \in E_{A}^{\infty} \text { and }|\omega \wedge \tau| \geq n\right\} .
$$

For every $\alpha>0$ let $\mathcal{K}_{\alpha}$ be the set of all complex-valued Hölder continuous (not necessarily bounded and allowing $-\infty$ with the convention that $e^{-\infty}=0$ and $\left.-\infty-(-\infty)=0\right)$ functions on $E_{A}^{\infty}$. Set

$$
\mathcal{K}_{\alpha}^{s}:=\left\{\rho \in \mathcal{K}_{\alpha}: \sum_{e \in E} \exp \left(\sup \left(\left.\operatorname{Re} \rho\right|_{[e]}\right)\right)<+\infty\right\} .
$$

An element of $\mathcal{K}_{\alpha}^{s}$ is called an $\alpha$-Hölder summable potential. Moreover, $\mathrm{H}_{\alpha}$ is defined to be the set of all bounded Hölder continuous functions. By endowing $\mathrm{H}_{\alpha}$ with the norm

$$
\|f\|_{\alpha}:=\|f\|_{\infty}+v_{\alpha}(f),
$$

the set $\mathrm{H}_{\alpha}$ becomes a complex Banach space. Also, the set $\mathrm{H}_{\alpha}$ forms a vector subspace of the Banach space $C_{b}:=C_{b}\left(E_{A}^{\infty}\right)$ of bounded continuous complex-valued functions defined on $E_{A}^{\infty}$. This Banach space is equipped with the supremum norm $\|\cdot\|_{\infty}$. We start with the following elementary observation.

Lemma 3.1. For every $R>0$ there exists $M=M_{R} \geq 1$ ( $M_{R}$ increases monotonically in $R$ ) such that if $|z-\xi| \leq R$, then $\left|\mathrm{e}^{\xi}-\mathrm{e}^{z}\right| \leq M \mathrm{e}^{\operatorname{Re} z}|z-\xi|$. 
Proof. Looking at the Taylor's series expansion of the exponential function about 0, we see that there exists a constant $M \geq 1$ such that $\left|e^{w}-1\right| \leq M|w|$, if $|w| \leq R$. Hence $\left|e^{\xi}-e^{z}\right|=\left|e^{z}\right|\left|e^{z-\xi}-1\right| \leq e^{\operatorname{Re} z} M|z-\xi|$.

An immediate application of the previous lemma gives rise to the following result.

Lemma 3.2. If $\rho \in \mathcal{K}_{\alpha}^{s}$, then $e^{\rho} \in \mathrm{H}_{\alpha}$ and $\left\|e^{\rho}\right\|_{\alpha} \leq\left(1+M_{v_{\alpha}(\rho)} v_{\alpha}(\rho)\right) e^{\sup (\operatorname{Re} \rho)}$.

Proof. It follows immediately from the definition of $\mathcal{K}_{\alpha}^{s}$ that $e^{\rho} \in C_{b}\left(E_{A}^{\infty}\right)$ and $\left\|e^{\rho}\right\|_{\infty} \leq$ $e^{\sup (\operatorname{Re} \rho)}$. Putting $R=v_{\alpha}(\rho)$, we get from Lemma 3.1 that if $\omega, \tau \in E_{A}^{\infty}$ and $\omega_{1}=\tau_{1}$, then

$$
\left|e^{\rho(\omega)}-e^{\rho(\tau)}\right| \leq M_{R} e^{\operatorname{Re}(\rho(\tau))}|\rho(\omega)-\rho(\tau)| \leq M_{R} e^{\sup (\operatorname{Re} \rho)} v_{\alpha}(\rho) \exp (-(|\omega \wedge \tau|-1)) .
$$

We are done.

Given $e \in E$ and $g: E_{A}^{\infty} \rightarrow \mathbb{C}$ define the mapping $g \circ e: E_{A}^{\infty} \rightarrow \mathbb{C}$ by the formula

$$
g \circ e(\omega)= \begin{cases}g(e \omega) & \text { if } A_{e \omega_{1}}=1 \\ -\infty & \text { if } A_{e \omega_{1}}=0\end{cases}
$$

if $g \in \mathcal{K}_{\alpha}^{s}$, and

$$
g \circ e(\omega)= \begin{cases}g(e \omega) & \text { if } A_{e \omega_{1}}=1 \\ 0 & \text { if } A_{e \omega_{1}}=0\end{cases}
$$

otherwise. As an immediate consequence of this definition, we have the following.

Lemma 3.3. If $g: E_{A}^{\infty} \rightarrow \mathbb{C}$ is a Hölder continuous function, then so is $g \circ e: E_{A}^{\infty} \rightarrow \mathbb{C}$ (for all $e \in E$ ) and $v_{\alpha}(g \circ e) \leq v_{\alpha}(g)$. If in addition, $g \in \mathrm{H}_{a}$, then also $g \circ e \in \mathrm{H}_{a}$ and $\|g \circ e\|_{\alpha} \leq\|g\|_{\alpha}$. If $g \in \mathcal{K}_{\alpha}^{s}$, then $\sup (\operatorname{Re}(g \circ e))=\sup \left(\operatorname{Re}\left(\left.g\right|_{[e]}\right)\right)$.

Let us prove the following.

Lemma 3.4. If $k, l \in \mathrm{H}_{\alpha}$, then $k l \in \mathrm{H}_{\alpha}$ and $\|k l\|_{\alpha} \leq 3\|k\|\left\|_{\alpha}\right\| l \|_{\alpha}$.

Proof. Obviously

$$
\|k l\|_{\infty} \leq\|k\|_{\infty}\|l\|_{\infty}
$$

Now fix $\omega, \tau \in E_{A}^{\infty}$ with $\omega_{1}=\tau_{1}$. Then

$$
\begin{aligned}
|k l(\omega)-k l(\tau)| & =|k(\omega)(l(\omega)-l(\tau))+l(\tau)(k(\omega)-k(\tau))| \\
& \leq|k(\omega)||l(\omega)-l(\tau)|+|l(\tau)||k(\omega)-k(\tau)| \\
& \leq\|k\|_{\infty} v_{\alpha}(l) \kappa^{|\omega \wedge \tau|}+|| l \|_{\infty} v_{\alpha}(k) \kappa^{|\omega \wedge \tau|} \\
& \leq 2|| k\left\|_{\alpha}|| l\right\|_{\alpha} \kappa^{|\omega \wedge \tau|} .
\end{aligned}
$$

Hence, $v_{\alpha}(k l) \leq 2\|k\|_{\alpha}|| l \|_{\alpha}$, and we complete the proof by combining this with (3.1) 
Now for every $\rho \in \mathcal{K}_{a}^{s}$ and every $e \in E$ define the operator $A_{\rho, e}: C_{b}\left(E_{A}^{\infty}\right) \rightarrow C_{b}\left(E_{A}^{\infty}\right)$ by the formula

$$
A_{\rho, e}(g)=e^{\rho \circ e} g \circ e .
$$

As an immediate consequence of Lemma 3.3, Lemma 3.2, Lemma 3.4 and the increasing property of the function $R \mapsto M_{R}$ as stated in Lemma 3.1, we get the following.

Lemma 3.5. If $\rho \in \mathcal{K}_{a}^{s}$ and $e \in E$, then the operator $A_{\rho, e}$ preserves the Banach space $\mathrm{H}_{\alpha}$. Moreover, we have that $\left\|A_{\rho, e}\right\|_{\alpha} \leq 3\left(1+M_{v_{\alpha}(\rho)} v_{\alpha}(\rho)\right) \exp \left(\sup \left(\operatorname{Re} \rho_{[e]}\right)\right)$.

Now notice that the function $v_{\alpha}$ is a pseudo-norm on the vector space $\mathcal{K}_{\alpha}$. So, it induces a pseudo-metric on $\mathcal{K}_{\alpha}\left((f, g) \mapsto v_{\alpha}(f-g)\right)$, and this pseudo-metric restricted to $\mathcal{K}_{\alpha}^{s}$ induces a topology on $\mathcal{K}_{\alpha}^{s}$, which in the sequel will be called the $\alpha$-Hölder topology. By $B_{\alpha}(\rho, r)=\left\{\theta \in \mathcal{K}_{\alpha}^{s}: v_{\alpha}(\theta-\rho)<r\right\}$ we denote the balls with respect to the pseudo-norm $v_{\alpha}$. Let $L\left(\mathrm{H}_{\alpha}\right)$ be the Banach space of all bounded linear operators acting on the Banach space $\mathrm{H}_{a}$. We shall prove the following.

Lemma 3.6. For every $e \in E$ the function $\rho \mapsto A_{\rho, e} \in L\left(\mathrm{H}_{\alpha}\right)$, defined on $\mathcal{K}_{\alpha}^{s}$, is continuous.

Proof. Note that $\mathcal{K}_{\alpha}^{s}$ is closed with respect to additions of functions and with respect to multiplication by scalars $\geq 1$. Fix $\rho \in \mathcal{K}_{\alpha}^{s}$ and consider an arbitrary $\theta \in B_{\alpha}(\rho, 1)$. Then, in view of, Lemma 3.5, we have that

$$
\left\|A_{\theta, e}-A_{\rho, e}\right\|_{\alpha}=\left\|A_{\theta-\rho, e}\right\|_{\alpha} \leq 3\left(1+M_{1}\right) \exp \left(\sup \left(\left.(\operatorname{Re} \theta-\operatorname{Re} \rho)\right|_{[e]}\right)\right) \leq 3\left(1+M_{1}\right) \exp \left(v_{\alpha}(\theta-\rho)\right) \text {. }
$$

Now, fix $\rho \in \mathcal{K}_{\alpha}^{s}$ and notice that for every $g \in C_{b}$, the function

$$
\mathcal{L}_{\rho}(g)=\sum_{e \in E} e^{\rho \circ e} g \circ e=\sum_{e \in E} A_{\rho, e}(g)
$$

is well-defined, it belongs to $C_{b}$ and $\left\|\mathcal{L}_{\rho}(g)\right\|_{\infty} \leq \sum_{e \in E} \exp \left(\sup \left(\left.\operatorname{Re} \rho\right|_{[e]}\right)\right)\|g\|_{\infty}$. The operator $\mathcal{L}_{\rho}$ acts continuously on $C_{b}$ with

$$
\left\|\mathcal{L}_{\rho}\right\|_{\infty} \leq \sum_{e \in E} \exp \left(\sup \left(\left.\operatorname{Re} \rho\right|_{[e]}\right)\right)<\infty .
$$

It follows from Lemma 3.5 that the operator $\mathcal{L}_{\rho}$ preserves the Banch space $\mathrm{H}_{\alpha}$ and,

$$
\left\|\mathcal{L}_{\rho}\right\|_{\alpha} \leq 3\left(1+M_{v_{\alpha}(\rho)} v_{\alpha}\right)(\rho) \sum_{e \in E} \exp \left(\sup \left(\left.\operatorname{Re} \rho\right|_{[e]}\right)\right) .
$$

Lemma 3.7. Suppose that $\Lambda$ is an open subset of the complex plane $\mathbb{C}$. Assume that

(a) for every $\lambda \in \Lambda$, we have that $\rho_{\lambda} \in \mathcal{K}_{\alpha}^{s}$,

(b) for every $\omega \in E_{A}^{\infty}$, the function $\Lambda \ni \lambda \mapsto \rho_{\lambda}(\omega) \in \mathbb{C}$, is holomorphic.

(c) the function $\Lambda \ni \lambda \mapsto \mathcal{L}_{\rho_{\lambda}} \in L\left(\mathrm{H}_{\alpha}\right)$ is continuous,

(d)

$$
V_{\alpha}(\Lambda):=\sup \left\{v_{\alpha}\left(\rho_{\lambda}\right): \lambda \in \Lambda\right\}<+\infty
$$


(e)

$$
\Sigma(\Lambda):=\sum_{e \in E} \exp \left(\sup \left\{\operatorname{Re} \rho_{\lambda} \circ e: \lambda \in \Lambda\right\}\right)<+\infty .
$$

Then the map $\Lambda \ni \lambda \mapsto \mathcal{L}_{\rho_{\lambda}} \in L\left(\mathrm{H}_{\alpha}\right)$ is holomorphic.

Proof. Let $\gamma \subset \Lambda$ be a simple closed, contractible in $\Lambda$, rectifiable curve. Fix $g \in \mathrm{H}_{\alpha}$ and $\omega \in E_{A}^{\infty}$. Since $V_{\alpha}(\Lambda)$ and $\Sigma(\Lambda)$ are both finite, it follows from Lemma 3.5 and the Weierstrass $M$-test that the series defining the function $\Lambda \ni \lambda \mapsto \mathcal{L}_{\rho_{\lambda}} g(\omega)$ converges absolutely uniformly in $\Lambda$. Therefore, the function $\Lambda \ni \lambda \mapsto \mathcal{L}_{\rho_{\lambda}} g(\omega) \in \mathbb{C}$ is holomorphic. Hence, by Cauchy's Theorem, $\int_{\gamma} \mathcal{L}_{\rho_{\lambda}} g(\omega) d \lambda=0$. Since the function $\Lambda \ni \lambda \mapsto \mathcal{L}_{\rho_{\lambda}} g \in$ $\mathrm{H}_{\alpha}$ is continuous, the integral $\int_{\gamma} \mathcal{L}_{\rho_{\lambda}} g(\omega) d \lambda$ exists, and for every $\omega \in E^{\infty}$, we have that $\int_{\gamma} \mathcal{L}_{\rho_{\lambda}} g d \lambda(\omega)=\int_{\gamma} \mathcal{L}_{\rho_{\lambda}} g(\omega) d \lambda=0$. Hence $\int_{\gamma} \mathcal{L}_{\rho_{\lambda}} g d \lambda=0$. Now, since the function $\lambda \mapsto$ $\mathcal{L}_{\rho_{\lambda}} \in L\left(\mathrm{H}_{\alpha}\right)$ is continuous, the integral $\int_{\gamma} \mathcal{L}_{\rho_{\lambda}} d \lambda$ exists, and for every $g \in \mathrm{H}_{\alpha}, \int_{\gamma} \mathcal{L}_{\rho_{\lambda}} d \lambda(g)=$ $\int_{\gamma} \mathcal{L}_{\rho_{\lambda}} g d \lambda=0$. Thus $\int_{\gamma} \mathcal{L}_{\rho_{\lambda}} d \lambda=0$ and, by Morera's theorem, the map $\Lambda \ni \lambda \mapsto \mathcal{L}_{\rho_{\lambda}} \in$ $L\left(\mathrm{H}_{\alpha}\right)$ is holomorphic. We are done.

The main result of this section is now concluded as follows.

Theorem 3.8. Suppose that $\Lambda$ is an open subset of $\mathbb{C}$ and that the function $\lambda \mapsto \rho_{\lambda} \in \mathcal{K}_{\alpha}^{s}$, $\lambda \in \Lambda$, is continuous. If the function $\lambda \mapsto \rho_{\lambda}(\omega) \in \mathbb{C}$, defined on $\Lambda$, is holomorphic for every $\omega \in E_{A}^{\infty}$, then the function $\lambda \mapsto \mathcal{L}_{\rho_{\lambda}} \in L\left(\mathrm{H}_{\alpha}\right)$ is also holomorphic.

Proof. Fix $\lambda_{0} \in \Lambda$. In view of Lemma 3.7 it suffices to show that there exists $\delta>0$ such that $V_{\alpha}\left(B\left(\lambda_{0}, \delta\right)\right)<+\infty, \Sigma\left(B\left(\lambda_{0}, \delta\right)\right)<+\infty$, and the function $B\left(\lambda_{0}, \delta\right) \ni \lambda \mapsto$ $\mathcal{L}_{\rho_{\lambda}} \in L\left(\mathrm{H}_{\alpha}\right)$ is continuous. Since, by the Weierstrass $M$-test and Lemma 3.5 along with Lemma 3.6, continuity of the function $B\left(\lambda_{0}, \delta\right) \ni \lambda \mapsto \mathcal{L}_{\rho_{\lambda}}$ follows from finiteness of the supremum $V_{\alpha}\left(B\left(\lambda_{0}, \delta\right)\right)$ and summability of the series $\Sigma\left(B\left(\lambda_{0}, \delta\right)\right)$, we only need to prove that $V_{\alpha}\left(B\left(\lambda_{0}, \delta\right)\right)<+\infty$ and $\Sigma\left(B\left(\lambda_{0}, \delta\right)\right)<+\infty$. Indeed, since the function $\Lambda \ni \lambda \mapsto$ $\rho_{\lambda} \in \mathcal{K}_{\alpha}^{s}$, is continuous, there exists $\delta>0$ so small that $v_{\alpha}\left(\rho_{\lambda}-\rho_{\lambda_{0}}\right)<1$ whenever $\left|\lambda-\lambda_{0}\right|<\delta$. We then have for all $\lambda \in B\left(\lambda_{0}, \delta\right)$ that $v_{\alpha}\left(\rho_{\lambda}\right) \leq \rho_{\lambda_{0}}+1$. Consequently, $V_{\alpha}\left(B\left(\lambda_{0}, \delta\right)\right) \leq \rho_{\lambda_{0}}+1$. Also, for all $\lambda \in B\left(\lambda_{0}, \delta\right)$ and all $a \in E$, we have that

$$
\exp \left(\sup \left(\left.\operatorname{Re} \rho_{\lambda}\right|_{[a]}\right)\right) \leq \exp \left(\sup \left(\left.\operatorname{Re} \rho_{\lambda_{0}}\right|_{[a]}\right)+1\right)=e \exp \left(\sup \left(\left.\operatorname{Re} \rho_{\lambda_{0}}\right|_{[a]}\right)\right) .
$$

Therefore, since $\lambda_{0} \in \mathcal{K}_{\alpha}^{s}$, we get

$$
\Sigma\left(B\left(\lambda_{0}, \delta\right)\right) \leq e \sum_{a \in E} \exp \left(\sup \left(\left.\operatorname{Re} \rho_{\lambda_{0}}\right|_{[a]}\right)\right)<\infty .
$$

We are done.

\section{Dimension analyticity in Graph Directed Markov Systems}

In this section we bring up the issue of real analyticity of the Hausdorff dimension function of a weakly regularly analytic family of strongly regular conformal graph directed Markov system. Our central idea is to embed the naturally arising, real-analytic family of PerronFrobenius operators into a family, which by applying Thorem 3.8 from the previous section, 
can be proven to be analytic. Then one uses the perturbation theory (Kato-Rellich Theorem) for linear operators, a version of Bowen's formula, and the Inverse Function Theorem to conclude the proof. Let $\Lambda \subset \mathbb{C}^{d}, d \geq 1$, be an open simply connected subset of $\mathbb{C}^{d}$. Let $\left\{\Phi_{\lambda}\right\}_{\lambda \in \Lambda}$ be a family of CGDMS with the same set $V$ of vertices, the same set $E$ of edges, the same finitely irreducible incidence matrix $A$ and the same seed pairs $\left\{\left(X_{v}, W_{v}\right)\right\}_{v \in V}$ with all $W_{w} \subset \mathbb{C}$. Fix $\lambda_{0} \in \Lambda$ and for every $\omega \in E_{A}^{\infty}$ consider the function $\psi_{\omega}: \Lambda \rightarrow \mathbb{C}$ given by the formula

$$
\psi_{\omega}(\lambda)=\frac{\left(\phi_{\omega_{1}}^{\lambda}\right)^{\prime}\left(\pi_{\lambda}(\sigma \omega)\right)}{\left(\phi_{\omega_{1}}^{\lambda_{0}}\right)^{\prime}\left(\pi_{\lambda_{0}}(\sigma \omega)\right)},
$$

where $\pi_{\lambda}=\pi_{\Phi_{\lambda}}: E_{A}^{\infty} \rightarrow X$ is the coding map induced by the CGDMS $\Phi_{\lambda}$. The family $\left\{\Phi_{\lambda}\right\}_{\lambda \in \Lambda}$ is said to be analytic if

(a) for every $e \in E$ and every $x \in X_{t(e)}$, the function $\lambda \mapsto \phi_{e}^{\lambda}(x), \lambda \in \Lambda$, is analytic.

The family $\left\{\Phi_{\lambda}\right\}_{\lambda \in \Lambda}$ is called weakly regular analytic if in addition the following hold.

(b) $\Phi^{\lambda_{0}}$ is strongly regular.

(c) There exist a function $\kappa: E \rightarrow(0,+\infty)$ and a constant $C_{1} \geq 1$ such that

$$
\left|\left(\phi_{\omega_{1}}^{\lambda}\right)^{\prime}\left(\pi_{\lambda}(\sigma(\omega))\right)\right| \leq C_{1} \exp \left(-\kappa\left(\omega_{1}\right)\right)
$$

for all $\lambda \in \Lambda$ and all $\omega \in E_{A}^{\infty}$.

In order to formulate our last condition required for weakly regular analyticity, we shall prove first the following.

Lemma 4.1. Suppose that $\left\{\Phi_{\lambda}\right\}_{\lambda \in \Lambda}$ is an analytic family of CGDMS. For every vertex $v \in V$ fix $x_{v} \in \operatorname{Int} X_{v}$. Then the family $\left\{\lambda \mapsto \phi_{\omega}^{\lambda}\left(x_{t(\omega)}\right)\right\}_{\omega \in E_{A}^{*}}$ consists of holomorphic maps on $\Lambda$ and this family is normal. Also, the family $\left\{\lambda \mapsto \pi_{\lambda}(\omega)\right\}_{\omega \in E_{A}^{\infty}}$ consists of holomorphic maps on $\Lambda$ and is normal.

Proof. Since all the maps $(\lambda, z) \mapsto \phi_{e}^{\lambda}(z),(\lambda, z) \in \Lambda \times \operatorname{Int} X_{t(e)}, e \in E$ are holomorphic, all the maps $\lambda \mapsto \phi_{\omega}^{\lambda}\left(x_{t(\omega)}\right), \omega \in E_{A}^{*}$ are also holomorphic. Since their ranges are all contained in the bounded set $\bigcup_{v \in V} \operatorname{Int} X_{v}$, the family $\left\{\lambda \mapsto \phi_{\omega}^{\lambda}\left(x_{t(\omega)}\right)\right\}_{\omega \in E_{A}^{*}}$ is normal. Therefore, since for every $\omega \in E_{A}^{\infty}$, the sequence of functions $\left(\lambda \mapsto \phi_{\left.\omega\right|_{n}}^{\lambda}\left(x_{t\left(\left.\omega\right|_{n}\right)}\right)\right)_{n=1}^{\infty}$ defined on $\Lambda$ converges pointwise to $\pi_{\lambda}(\omega)$, we conclude that each function $\lambda \mapsto \pi_{\lambda}(\omega)$, defined on $\Lambda$, is holomorphic. Since the range of all these functions is in the bounded set $\bigcup_{v \in V} X_{v}$, the family $\left\{\lambda \mapsto \pi_{\lambda}(\omega)\right\}_{\omega \in E_{A}^{\infty}}$ is normal. We are done.

As an immediate consequence of this lemma, item (a) above, and definition (4.1), we get that for every $\omega \in E_{A}^{\infty}$, the function $\psi_{\omega}: \Lambda \rightarrow \mathbb{C}$ is analytic. Now we can complete the definition of weak regular analyticity by giving the following final condition.

(d) For every $\omega \in E_{A}^{\infty}$ let $\log \psi_{\omega}: \Lambda \rightarrow \mathbb{C}$ the the holomorphic branch of logarithm of $\psi_{\omega}$ normalised so that $\log \psi_{\omega}\left(\lambda_{0}\right)=0$. We demand that the family of functions

$$
\left\{\lambda \mapsto \frac{1}{\kappa\left(\omega_{1}\right)} \log \psi_{\omega}(\lambda)\right\}_{\omega \in E_{A}^{\infty}}
$$


is bounded and, consequently, normal.

Let $h_{\lambda}=\operatorname{HD}\left(J_{\Phi^{\lambda}}\right)$ be the Hausdorff dimension of the limit set of the CGDMSs $\Phi^{\lambda}$. The goal of this section is to prove the following.

Theorem 4.2. If $\left\{\Phi^{\lambda}\right\}_{\lambda \in \Lambda}$ is a weakly regularly analytic family of CGDMS, then the function $\lambda \mapsto h_{\lambda}=\operatorname{HD}\left(J_{\Phi^{\lambda}}\right)$ is real-analytic throughout $\Lambda$.

This theorem was formulated in [10] only for iterated function systems and, what is more important, under the assumption that the function $\kappa$ is constant. There was no proof in [10] but only a one-line indication of how to form the proof based on [16] and [15]. Concluding, since this theorem is central for us in the current paper, since it is of interest itself, and since there is no written proof, even of its earlier weaker version, we have decided to provide here a self-contained proof of this theorem (Theorem 4.2). The general strategy of the proof is based on Theorem 3.8 from the previous section. We start it as follows. For every $z=\left(z_{1}, z_{2}, \ldots, z_{d}\right) \in \mathbb{C}^{d}$ and $r_{1}, r_{2}, \ldots, r_{d}>0$ let

$$
D_{d}\left(z ; r_{1}, r_{2}, \ldots, r_{d}\right)=\left\{\left(w_{1}, w_{2}, \ldots, w_{d}\right) \in \mathbb{C}^{d}:\left|w_{j}-z_{j}\right|<r_{j} \text { for all } 1 \leq j \leq d\right\} .
$$

In the case when all radii $r_{j}$ are equal, say to $r$, we will frequently write shortly $D_{d}(z ; r)$ for $D_{d}(z ; r, r, \ldots, r)$. Fix $r>0$ so small that $D_{d}\left(\lambda_{0} ; r\right) \subset \Lambda$. For ease of exposition we assume now that $d=1$, i.e. that $\Lambda$ is an open subset of the complex plane $\mathbb{C}$. Because of item (d) above, for every $\omega \in E^{\infty}$, the function $\log \psi_{\omega}$ expands in its Taylor series on $D_{1}\left(\lambda_{0} ; r\right)$ :

$$
\log \psi_{\omega}(\lambda)=\sum_{n=0}^{\infty} a_{n}(\omega)\left(\lambda-\lambda_{0}\right)^{n}
$$

and, by item (d) again, there exists a constant $M_{1}>0$, independent of $\lambda \in \Lambda$ and $\omega \in E_{A}^{\infty}$, such that

$$
\left|\log \psi_{\omega}(\lambda)\right| \leq M_{1} \kappa\left(\omega_{1}\right) .
$$

Hence, applying Cauchy's estimates, we get for every $n \geq 0$ that

$$
\left|a_{n}(\omega)\right| \leq \frac{M_{1} \kappa\left(\omega_{1}\right)}{r^{n}} .
$$

For every $\lambda=x+i y \in D\left(\lambda_{0} ; r\right)$, we have from (4.2) that

$$
\begin{aligned}
\operatorname{Re} \log \psi_{\omega}(\lambda) & =\sum_{p, q=0}^{\infty} \operatorname{Re}\left(a_{p+q}(\omega)\left(\begin{array}{c}
p+q \\
q
\end{array}\right) i^{q}\right)\left(x-\operatorname{Re} \lambda_{0}\right)^{p}\left(y-\operatorname{Im} \lambda_{0}\right)^{q} \\
& =\sum_{p, q=0}^{\infty} c_{p+q}(\omega)\left(\begin{array}{c}
p+q \\
q
\end{array}\right) i^{q}\left(x-\operatorname{Re} \lambda_{0}\right)^{p}\left(y-\operatorname{Im} \lambda_{0}\right)^{q}
\end{aligned}
$$

where, due to (4.4),

$$
\left|c_{p+q}(\omega)\right| \leq\left(\begin{array}{c}
p+q \\
q
\end{array}\right)\left|a_{p+q}(\omega)\right| \leq 2^{p+q}\left|a_{p+q}(\omega)\right| \leq 2^{p+q} M_{1} \kappa\left(\omega_{1}\right) r^{-(p+q)} .
$$


Hence, employing the embedding $\mathbb{C} \rightarrow \mathbb{C}^{2}=\mathbb{C} \times \mathbb{C}, x+i y \mapsto(x, y)$, we see that $\operatorname{Re} \log \psi_{\omega}$ extends by the same power series expansion $\sum_{p, q=0}^{\infty} c_{p+q}(\omega)\left(\begin{array}{c}p+q \\ q\end{array}\right) i^{q}\left(x-\operatorname{Re} \lambda_{0}\right)^{p}\left(y-\operatorname{Im} \lambda_{0}\right)^{q}$, $(x, y) \in \mathbb{C}^{2}$, to a complex-valued analytic function on the polydisk $D_{2}\left(\lambda_{0} ; r / 4\right)$. Keep the same symbol Re $\log \psi_{\omega}$ for this extension and note that

$$
\left|\operatorname{Re} \log \psi_{\omega}\right| \leq 4 M_{1} \kappa\left(\omega_{1}\right) \text { on } D_{2}\left(\lambda_{0} ; r / 4\right) \text {. }
$$

Define the potential $\zeta_{\omega}: D_{2}\left(\lambda_{0} ; r / 4\right) \rightarrow \mathbb{C}$ by the formula

$$
\zeta_{\omega}(\lambda)=\operatorname{Re} \log \psi_{\omega}(\lambda)+\log \left|\left(\phi_{\omega_{1}}^{\lambda_{0}}\right)^{\prime}\left(\pi_{\lambda_{0}}(\sigma \omega)\right)\right| .
$$

Fix $t_{0}>\theta\left(\Phi^{\lambda_{0}}\right)$ and put

$$
\bar{r}=\min \left\{r / 4,\left(t_{0}-\theta\left(\Phi^{\lambda_{0}}\right)\right) / 2\right\} .
$$

Note that for all $(\lambda, t) \in D_{3}\left(\left(\lambda_{0}, t_{0}\right) ; \bar{r}\right):=\left(D_{2}\left(\lambda_{0} ; \bar{r}\right) \cap \mathbb{R}^{2}\right) \times\left(D_{1}\left(t_{0}, \bar{r}\right) \cap \mathbb{R}\right)$, we have by (4.1) that

$$
\exp \left(t \zeta_{\omega}(\lambda)\right)=\left|\psi_{\omega}(\lambda)\right|^{t}\left|\left(\phi_{\omega_{1}}^{\lambda_{0}}\right)^{\prime}\left(\pi_{\lambda_{0}}(\sigma \omega)\right)\right|^{t}=\left|\left(\phi_{\omega_{1}}^{\lambda}\right)^{\prime}\left(\pi_{\lambda}(\sigma \omega)\right)\right|^{t} .
$$

Now, our goal is to prove the following result announced at the begining of this section.

Lemma 4.3. There exists $r_{2} \in(0, \bar{r})$ such that the family of potentials

$$
(\lambda, t) \mapsto t \zeta_{(\cdot)}(\lambda): E_{A}^{\infty} \rightarrow \mathbb{C},(\lambda, t) \in D_{3}\left(\left(\lambda_{0}, t_{0}\right) ; \bar{r}\right),
$$

satisfies the hypothesis of Theorem 3.8.

Proof. Obviously, for every $\omega \in E_{A}^{\infty}$, the function $(\lambda, t) \mapsto t \zeta_{\omega}(\lambda),(\lambda, t) \in D_{3}\left(\left(\lambda_{0}, t_{0}\right) ; \bar{r}\right)$ is holomorphic. Using (4.6) we get for all $\omega \in E_{A}^{\infty}$ and all $(\lambda, t) \in D_{3}\left(\left(\lambda_{0}, t_{0}\right) ; \bar{r}\right)$ that

$$
\begin{aligned}
\left|\exp \left(t \zeta_{\omega}(\lambda)\right)\right| & =\exp \left(\operatorname{Re}\left(t \operatorname{Re} \log \psi_{\omega}(\lambda)+t \log \left|\left(\phi_{\omega_{1}}^{\lambda_{0}}\right)^{\prime}\left(\pi_{\lambda_{0}}(\sigma \omega)\right)\right|\right)\right) \\
& =\exp \left(\operatorname{Re}\left(t \operatorname{Re} \log \psi_{\omega}(\lambda)\right)\right)\left|\left(\phi_{\omega_{1}}^{\lambda_{0}}\right)^{\prime}\left(\pi_{\lambda_{0}}(\sigma \omega)\right)\right|^{\operatorname{Re} t} \\
& \leq \exp \left(|t|\left|\operatorname{Re} \log \psi_{\omega}(\lambda)\right|\right)\left|\left(\phi_{\omega_{1}}^{\lambda_{0}}\right)^{\prime}\left(\pi_{\lambda_{0}}(\sigma \omega)\right)\right|^{\operatorname{Re} t} \\
& \leq \exp \left(4 M_{1} \kappa\left(\omega_{1}\right)|t|\right)\left|\left(\phi_{\omega_{1}}^{\lambda_{0}}\right)^{\prime}\left(\pi_{\lambda_{0}}(\sigma \omega)\right)\right|^{\operatorname{Re} t} \\
& \leq \exp \left(4 M_{1}\left(t_{0}+\bar{r}\right) \kappa\left(\omega_{1}\right)\right)\left|\left(\phi_{\omega_{1}}^{\lambda_{0}}\right)^{\prime}\left(\pi_{\lambda_{0}}(\sigma \omega)\right)\right|^{\theta\left(\Phi^{\lambda_{0}}\right)+\bar{r}} .
\end{aligned}
$$

Now, it follows from item (d) of weakly regular analyticity (equicontinuity and $\log \psi_{\omega}\left(\lambda_{0}\right)=$ $0)$ that if $r_{2} \in(0, \bar{r})$ is sufficiently small, and $\lambda$ appearing in formula (4.3) belongs to the disk $D_{1}\left(\lambda_{0} ; r_{2}\right)$, then we can have $M_{1}>0$ as small as we wish, for example

$$
M_{1} \leq \frac{1}{8} \bar{r}\left(t_{0}+\bar{r}\right)^{-1}
$$

Inserting this inequality into (4.8) and using condition (c), we get

$$
\left|\exp \left(t \zeta_{\omega}(\lambda)\right)\right| \leq C_{1}^{(\bar{r} / 2)}\left|\left(\phi_{\omega_{1}}^{\lambda_{0}}\right)^{\prime}\left(\pi_{\lambda_{0}}(\sigma \omega)\right)\right|^{\theta\left(\Phi^{\lambda_{0}}\right)+\frac{\bar{r}}{2}}
$$

for all $\omega \in E^{\infty}$ and all $(\lambda, t) \in D_{3}\left(\left(\lambda_{0}, t_{0}\right) ; r_{2}\right)$. Therefore,

$$
\sum_{e \in E}\left\|\exp \left(\left.t \zeta\right|_{[e]}\right)\right\|_{\infty} \leq C_{1}^{(\bar{r} / 2)} \sum_{e \in E}\left\|\left(\phi_{e}^{\lambda_{0}}\right)^{\prime}\right\|^{\theta\left(\Phi^{\lambda_{0}}\right)+\frac{\bar{r}}{2}}<+\infty .
$$


Hence, for every $(\lambda, t) \in D_{3}\left(\left(\lambda_{0}, t_{0}\right) ; r_{2}\right)$, we have that $t \zeta_{(\cdot)}(\lambda) \in \mathcal{K}_{\alpha}^{s}$. So, in order to prove our lemma we only need to show that for every $(\lambda, t) \in D_{3}\left(\left(\lambda_{0}, t_{0}\right) ; r_{2}\right)$, the function $\omega \mapsto$ $\left.t \zeta_{\omega}(\lambda)\right), \omega \in E^{\infty}$, is Hölder continuous, and then that the mapping $(\lambda, t) \mapsto t \zeta_{(\cdot)}(\lambda) \in \mathcal{K}_{\alpha}^{s}$ is continuous. Applying Lemma 4.2.2 from [7] and Koebe's Distortion Theorem for argument (see Corollary on page 353 of [1]), we conclude that there exists a constant $K_{1} \geq 1$ such that $\left|\log \left(\phi_{\omega}^{\lambda}\right)^{\prime}(y)\right|-\log \left(\phi_{\omega}^{\lambda}\right)^{\prime}(x)\left|\leq K_{1}\right| y-x \mid$ for all $\lambda \in \Lambda$, all $\omega \in E_{A}^{\infty}$ and all $x, y \in X_{t(\omega)}$. Hence if $\omega, \tau \in E_{A}^{\infty}$ and $|\omega \wedge \tau| \geq 1$, then

$$
\begin{aligned}
\left|\log \psi_{\omega}(\lambda)-\log \psi_{\tau}(\lambda)\right| & =\left|\log \left(\left(\phi_{\omega_{1}}^{\lambda}\right)^{\prime}\left(\pi_{\lambda}(\sigma \omega)\right)\right)-\log \left(\left(\phi_{\tau_{1}}^{\lambda}\right)^{\prime}\left(\pi_{\lambda}(\sigma \tau)\right)\right)\right| \\
& \leq K_{1}\left|\pi_{\lambda}(\sigma \omega)-\pi_{\lambda}(\sigma \tau)\right| \leq K_{1} s^{|\omega \wedge \tau|-1} \operatorname{diam}(X) \\
& =K_{1} s^{-1} s^{|\omega \wedge \tau|} \operatorname{diam}(X) .
\end{aligned}
$$

Thus, using Cauchy's Estimates again, we conclude that for all $n \geq 0$,

$$
\left|a_{n}(\omega)-a_{n}(\tau)\right| \leq K_{1} s^{-1} \operatorname{diam}(X) s^{|\omega \wedge \tau|} r^{-n}
$$

Consequently,

$$
\left|c_{p, q}(\omega)-c_{p, q}(\tau)\right| \leq 2^{p+q}\left|a_{p+q}(\omega)-a_{p+q}(\tau)\right| \leq 2^{p+q} K_{1} s^{-1} \operatorname{diam}(X) r^{-(p+q)} s^{|\omega \wedge \tau|} .
$$

Therefore,

$$
\left|\operatorname{Re} \log \psi_{\omega}(\lambda)-\operatorname{Re} \log \psi_{\tau}(\lambda)\right| \leq 4 K_{1} s^{-1} \operatorname{diam}(X) s^{|\omega \wedge \tau|}
$$

for all $\lambda \in D_{2}\left(\lambda_{0}, r / 2\right)$ and $\omega, \tau \in E_{A}^{\infty}$ with $|\omega \wedge \tau| \geq 1$. Hence, using also (4.9), we conclude that

$$
\left|t \zeta_{\omega}(\lambda)-t \zeta_{\tau}(\lambda)\right| \leq 5 K_{1} s^{-1} \operatorname{diam}(X) s^{|\omega \wedge \tau|}
$$

for all $(\lambda, t) \in D_{3}\left(\left(\lambda_{0}, t_{0}\right) ; r_{2}\right)$ and all $\omega, \tau \in E_{A}^{\infty}$ with $|\omega \wedge \tau| \geq 1$. The proof that the function $\left.\omega \mapsto t \zeta_{\omega}(\lambda)\right)$ belongs to $\mathcal{K}_{a}^{s}$ is complete. This function is obviously continuous on the polydisk $D_{3}\left(\left(\lambda_{0}, t_{0}\right) ; r_{2}\right)$ with respect to the variable $t$. It is therefore sufficient to prove Lipschitz continuity of the functions $\lambda \mapsto t \zeta_{(\cdot)}(\lambda) \in \mathcal{K}_{\alpha}^{s}$ with Lipschitz constants independent of $t$. In order to do it, fix $\lambda=\left(\lambda_{x}, \lambda_{y}\right)$ and $\lambda^{\prime}=\left(\lambda_{x}^{\prime}, \lambda_{y}^{\prime}\right)$ in $D_{2}\left(\lambda_{0}, r_{2}\right)$. Put $a_{x}=\lambda_{x}^{\prime}-\operatorname{Re} \lambda_{0}, a_{y}=\lambda_{y}^{\prime}-\operatorname{Im} \lambda_{0}, b_{x}=\lambda_{x}-\operatorname{Re} \lambda_{0}$ and $b_{y}=\lambda_{y}-\operatorname{Im} \lambda_{0}$. We then have

$$
\begin{aligned}
\left|a_{x}^{p} a_{y}^{q}-b_{x}^{p} b_{y}^{q}\right| & =\left|a_{x}^{p}\left(a_{y}^{q}-b_{y}^{q}\right)+b_{y}^{q}\left(a_{x}^{p}-b_{x}^{p}\right)\right| \\
& \leq\left|a_{x}^{p}\right|\left|a_{y}-b_{y}\right| \sum_{i=0}^{q-1}\left|a_{y}\right|^{i}\left|b_{y}\right|^{q-1-i}+\left|b_{y}^{q}\right|\left|a_{x}-b_{x}\right| \sum_{i=0}^{p-1}\left|a_{x}\right|^{i}\left|b_{x}\right|^{p-1-i} \\
& \leq\left(q\left(\frac{r}{4}\right)^{p}\left(\frac{r}{4}\right)^{q-1}+p\left(\frac{r}{4}\right)^{q}\left(\frac{r}{4}\right)^{p-1}\right)\left\|\lambda^{\prime}-\lambda\right\| \\
& \leq \frac{4}{r}(p+q)\left(\frac{r}{4}\right)^{p}\left(\frac{r}{4}\right)^{q}\left\|\lambda^{\prime}-\lambda\right\| .
\end{aligned}
$$


Now fix $\omega, \tau \in E_{A}^{\infty}$ with $\omega_{1}=\tau_{1}$. It follows from (4.5), (4.9) and (4.10) that

$$
\begin{aligned}
& \left|\operatorname{Re} \log \psi_{\omega}\left(\lambda^{\prime}\right)-\operatorname{Re} \log \psi_{\omega}(\lambda)-\left(\operatorname{Re} \log \psi_{\tau}\left(\lambda^{\prime}\right)-\operatorname{Re} \log \psi_{\tau}(\lambda)\right)\right| \\
& =\left|\sum_{p, q=0}^{\infty}\left(c_{p, q}(\omega)-c_{p, q}(\tau)\right)\left(\left(\lambda_{x}^{\prime}-\operatorname{Re} \lambda_{0}\right)^{p}\left(\lambda_{y}^{\prime}-\operatorname{Im} \lambda_{0}\right)^{q}-\left(\lambda_{x}-\operatorname{Re} \lambda_{0}\right)^{p}\left(\lambda_{y}-\operatorname{Im} \lambda_{0}\right)^{q}\right)\right| \\
& \leq 4 K_{1} s^{-1} \operatorname{diam}(X) r^{-1} s^{|\omega \wedge \tau|}\left|\lambda^{\prime}-\lambda\right| \sum_{p, q=0}^{\infty}(p+q) 2^{-(p+q)} \\
& =C\left|\lambda^{\prime}-\lambda\right| s^{|\omega \wedge \tau|}
\end{aligned}
$$

where $C=4 K_{1} s^{-1} \operatorname{diam}(X) r^{-1} \sum_{p, q=0}^{\infty}(p+q) 2^{-(p+q)}$ is finite. Thus,

$$
\left.v_{\alpha}\left(t \operatorname{Re} \log \psi_{(\cdot)}\left(\lambda^{\prime}\right)-t \operatorname{Re} \log \psi_{(\cdot)}(\lambda)\right)\right) \leq C\left(\left|t_{0}\right|+r_{2}\right) r^{-1}\left|\lambda^{\prime}-\lambda\right| .
$$

for all $(\lambda, t) \in D_{3}\left(\left(\lambda_{0}, t_{0}\right) ; r_{2}\right)$. Since $t \zeta_{(\cdot)}\left(\lambda^{\prime}\right)-t \zeta_{(\cdot)}(\lambda)=t \operatorname{Re} \log \psi_{(\cdot)}\left(\lambda^{\prime}\right)-t \operatorname{Re} \log \psi_{(\cdot)}(\lambda)$, the proof of continuity of the function $(\lambda, t) \mapsto t \zeta_{(\cdot)}(\lambda) \in \mathcal{K}_{\alpha}^{s},(\lambda, t) \in D_{3}\left(\left(\lambda_{0}, t_{0}\right) ; r_{2}\right)$, is complete, and the proof of Lemma 4.3 is finished.

For every $(\lambda, t) \in D_{3}\left(\left(\lambda_{0}, h_{\lambda_{0}}\right) ; r_{2}\right)$ put

$$
\mathcal{L}_{\lambda, t}=\mathcal{L}_{t \zeta_{(\cdot)}}(\lambda): \mathrm{H}_{\alpha} \rightarrow \mathrm{H}_{\alpha}
$$

The number $h_{\lambda_{0}}$ is larger than $\theta\left(\Phi^{\lambda_{0}}\right)$ because of strong regularity of the system $\Phi^{\lambda_{0}}$. In view of (4.7) and Theorem 2.4.6 from [7], $e^{\mathrm{P}_{\lambda_{0}}\left(h_{\lambda_{0}}\right)}$ is a simple isolated eigenvalue of the operator $\mathcal{L}_{\lambda_{0}, h_{\lambda_{0}}}: \mathrm{H}_{\alpha} \rightarrow \mathrm{H}_{\alpha}$. Hence, in view of Lemma 4.3 and Theorem 3.8, Kato-Rellich Perturbation Theorem ([2], Theorem XII.8) is applicable to yield a number $r_{3} \in\left(0, r_{2}\right]$ and a holomorphic function $\gamma: D_{3}\left(\left(\lambda_{0}, h_{\lambda_{0}}\right) ; r_{3}\right) \rightarrow \mathbb{C}$ such that $\gamma\left(\lambda_{0}, h_{\lambda_{0}}\right)=e^{\mathrm{P}_{\lambda_{0}}\left(h_{\lambda_{0}}\right)}$, and for all $(\lambda, t) \in D_{3}\left(\left(\lambda_{0}, h_{\lambda_{0}}\right) ; r_{3}\right), \gamma(\lambda, t)$ is a simple isolated eigenvalue of the operator $\mathcal{L}_{\lambda, t}: \mathrm{H}_{\alpha} \rightarrow \mathrm{H}_{\alpha}$, with the remainder of the spectrum uniformly separated from $\gamma(\lambda, t)$. In particular there exists $r_{4} \in\left(0, r_{3}\right]$ and $\eta>0$ such that

$$
\sigma\left(\mathcal{L}_{\lambda, t}\right) \cap D_{1}\left(e^{\mathrm{P}_{\lambda_{0}}\left(h_{\lambda_{0}}\right)}, \eta\right)=\{\gamma(\lambda, t)\}
$$

for all $(\lambda, t) \in D_{3}\left(\left(\lambda_{0}, h_{\lambda_{0}}\right) ; r_{4}\right)$. Now, $e^{\mathrm{P}_{\lambda}(t)}$ is the spectral radius $r\left(\mathcal{L}_{\lambda, h_{\lambda}}\right)$ of the operator $\mathcal{L}_{\lambda, t}$ for all $(\lambda, t) \in B\left(\lambda_{0}, r_{4}\right) \times B\left(h_{\lambda_{0}}, r_{4}\right)$. Setting $t=h_{\lambda_{0}}$, in view of semi-continuity of the spectral set function (see Theorem 10.20 on p.256 in [11]), taking $r_{4}$ appropriately smaller, we will also have that $r\left(\mathcal{L}_{\lambda, t}\right) \in\left[0, e^{\mathrm{P}_{\lambda_{0}}\left(h_{\lambda_{0}}\right)}+\eta\right)$. Along with (4.11), these facts imply that $e^{\mathrm{P}_{\lambda}(t)}=\gamma(\lambda, t)$ for all $(\lambda, t) \in B\left(\lambda_{0}, r_{4}\right) \times B\left(t_{0}, r_{4}\right)$. Consequently, the function $(\lambda, t) \mapsto \mathrm{P}_{\lambda}(t),(\lambda, t) \in B\left(\lambda_{0}, r_{4}\right) \times B\left(t_{0}, r_{4}\right)$ is real-analytic. By Bowen's formula (see Theorem 2.3) and strong regularity of the system $\Phi^{\lambda_{0}}$, we get that $\mathrm{P}_{\lambda_{0}}\left(h_{\lambda_{0}}\right)=0$. But, by Proposition 2.6.13 and Proposition 3.1.4 from [7],

$$
\left.\frac{\partial \mathrm{P}}{\partial t}\right|_{\left(\lambda_{0}, h_{\lambda_{0}}\right)}=\int \log \left|\left(\phi_{\omega_{1}}^{\lambda_{0}}\right)\left(\pi_{\lambda_{0}}(\sigma(\omega))\right)\right| d \mu_{0}(\omega)<0,
$$

where $\mu_{0}$ is the Gibbs (equilibrium) state of the potential $\omega \mapsto h_{\lambda_{0}} \log \left|\left(\phi_{\omega_{1}}^{\lambda_{0}}\right)\left(\pi_{\lambda_{0}}(\sigma(\omega))\right)\right|$ (see [7] for these concepts in the context of graph directed Markov systems). Consequently, 
it follows from the Implicit Function Theorem that there exist $r_{5} \in\left(0, r_{4}\right]$ and a realanalytic function $t(\lambda), \lambda \in B\left(\lambda_{0}, r_{5}\right)$ such that $\mathrm{P}_{\lambda}(t(\lambda))=0$ and $t\left(\lambda_{0}\right)=h_{\lambda_{0}}$. Invoking Theorem 2.3 again, we conclude that $h_{\lambda}=t(\lambda)$, and the proof of Theorem 4.2 is finished.

\section{Generalized Polynomial-Like Mappings; basics}

In this section we recall from [13] and [14] the class of semihyperbolic generalized polynomiallike mappings (GPL) and canonically associate to them conformal graph directed Markov systems in the sense of [7]. For $U \subset \mathbb{C}$, an open Jordan domain with smooth boundary, let $\mathcal{U}:=\bigcup_{i \in I} U_{i}$ be a finite union of open Jordan domains $U_{i}$ whose closures are pairwise disjoint and are all contained in $U$. A GPL-map $f$ is a holomorphic map

$$
f: \mathcal{U} \rightarrow U
$$

such that for each $i \in I$ the restriction of $f$ to $U_{i}$ is a surjective branched covering map having at most one critical (branching) point. The Julia set $J(f)$ of $f$ is defined to be the set of all those points $z$ in $\mathcal{U}$ such that all iterates of $z$ under $f$ are well-defined but each neighborhood of $z$ has a point which is eventually mapped out of $\mathcal{U}$. Also, define

$$
\operatorname{Crit}(f):=\left\{c: f^{\prime}(c)=0\right\} \quad \text { and } \operatorname{Crit}(J(f)):=J(f) \cap \operatorname{Crit}(f) .
$$

The index set $I$ is split in the following way.

$$
\begin{aligned}
& I_{o}:=\left\{i \in I: \overline{U_{i}} \cap \overline{\bigcup_{n \geq 1} f^{n}(\operatorname{Crit}(f))}=\emptyset\right\} \quad \text { ('post-critical free indices'), } \\
& I_{c}:=\left\{i \in I: U_{i} \cap \operatorname{Crit}(f) \neq \emptyset\right\} \quad \text { ('critical indices'), } \\
& I_{r}:=I \backslash I_{c} \quad \text { ('regular indices'). }
\end{aligned}
$$

With this decomposition of the finite index set $I$, we put

$$
\mathcal{U}_{o}:=\bigcup_{i \in I_{o}} U_{i}, \mathcal{U}_{c}:=\bigcup_{i \in I_{c}} U_{i}, \mathcal{U}_{r}:=\bigcup_{i \in I_{r}} U_{i} .
$$

For every $i \in I_{r}$ set

$$
f_{i}^{-1}:=\left(\left.f\right|_{U_{i}}\right)^{-1}: U \rightarrow U_{i}
$$

Definition 5.1. A GPL-map $f$ is called semihyperbolic if and only if $I_{c} \subset I_{o}$.

Throughout the paper we assume $f$ to be a semihyperbolic GPL-map. The following lemma is immediate.

Lemma 5.2. If $f$ is a semihyperbolic GPL-map $f$, then the closure of the forward orbit of Crit $(f)$ is nowhere dense in $J(f)$.

We now associate to the semihyperbolic map $f$ a graph directed Markov system. 
Proposition 5.3. Let $f$ be a semihyperbolic GPL-map. Then there exists a finitely primitive order 1 conformal GDMS $\Phi_{f}$ with $J_{\Phi_{f}} \subset J(f)$ such that

$$
J_{\Phi_{f}} \cap \mathcal{U}_{o}=J(f) \cap \mathcal{U}_{o} \backslash \bigcup_{n \geq 0} f^{-n}\left(\bigcap_{k \geq 0} f^{-k}\left(\mathcal{U}_{r}\right)\right) .
$$

In addition $\operatorname{HD}\left(J_{\Phi_{f}}\right)=\operatorname{HD}(J(f))$.

Proof. We take $I_{o}$ to be the set of vertices. The conformal univalent contractions of our system are defined as follows. For every $i \in I_{o}$ fix an open topological disk $\tilde{U}_{i}$ with smooth boundary which contains $U_{i}$ and whose closure is disjoint from the closure of the postcritical set $\bigcup_{n \geq 1} f^{n}(\operatorname{Crit}(f))$. Of course we can always take $U_{i}$ for $\tilde{U}_{i}$ but we will need in the next section such a larger choice. By definition of the sets $\tilde{U}_{i}$, for each vertex $i \in I_{o}$ all the holomorphic inverse branches of any iterate of $f$ are well-defined on a fixed neighborhood $W_{i}$ of the closure of $\tilde{U}_{i}$. Hence, for each $j \in I_{o}$ and $n \geq 1$, we consider all the holomorphic inverse branches $f_{*}^{-n}: \tilde{U}_{j} \rightarrow U$ of $f^{n}$ such that $f_{*}^{-n}\left(\tilde{U}_{j}\right) \subset \tilde{U}_{k}$ for some $k \in I_{o}$ and $f^{i}\left(f_{*}^{-n}\left(\tilde{U}_{j}\right)\right) \cap \mathcal{U}_{o}=\emptyset$ for all $i=1,2, \ldots, n-1$. We then write $\phi_{e}: \tilde{U}_{t(e)} \rightarrow \tilde{U}_{i(e)}$ for $f_{*}^{-n}: \tilde{U}_{j} \rightarrow \tilde{U}_{k}$, where $t(e)=j$ and $i(e)=k$. Also, we define $\|e\|:=n$. Now, let

$$
\Phi^{f}:=\left\{\phi_{e}: \overline{\tilde{U}_{t(e)}} \rightarrow \overline{\tilde{U}_{i(e)}}\right\}_{e \in E_{f}}
$$

where $E_{f}$ is some countable auxiliary set parametrizing the family $\Phi^{f}$. Note that the set $I_{o}$ of vertices is finite, whereas in general the set $E_{f}$ of edges is infinite. We immediately obtain from the construction of $\Phi_{f}$ that

$$
J_{\Phi^{f}} \cap \mathcal{U}_{o}=J(f) \cap \mathcal{U}_{o} \backslash \bigcup_{n \geq 0} f^{-n}\left(\bigcap_{k \geq 0} f^{-k}\left(\mathcal{U}_{r}\right)\right)
$$

and the limit set $J_{\Phi^{f}}$ is independent of the admissible choice of the disks $\tilde{U}_{j}, j \in I_{o}$. We remark that the cone condition is satisfied, since for each $i \in I_{0}$ the boundaries of the disks $\overline{\tilde{U}}_{i}$ are smooth. Also, the open set condition follows immediately from the construction of $\Phi^{f}$, noting that the elements of $\Phi^{f}$ are inverse branches of forward iterates of $f$. Finally, since for each pair $j, k \in I_{o}$ there is a holomorphic inverse $f_{i, k}^{-1}$ of $f$ defined on $\tilde{U}_{k}$ and mapping $\tilde{U}_{k}$ into $\tilde{U}_{j}$ and since $f_{i, k}^{-1}: \tilde{U}_{k} \rightarrow \tilde{U}_{j}$ is in $\Phi_{f}$, we conclude that the system $\Phi_{f}$ is primitive of order 1 . We are left to show equality of dimensions. Indeed, it follows from Theorem 2.1 in [13] and continuity of the pressure function $\mathrm{P}_{f}(t)$ proved there (by the item (6) of the definition of the pressure function in [13], this function is convex and hence continuous) that $\mathrm{P}_{f}(\mathrm{HD}(J(f))=0$. It then follows from Lemma 4.6 in [13] that $\mathrm{P}_{\Phi_{f}}\left(\mathrm{HD}(J(f))=0\right.$. Consequently, by Theorem 2.3, it follows that $\operatorname{HD}\left(J_{\Phi_{f}}\right)=\operatorname{HD}(J(f))$.

Denote the corresponding incidence matrix by $A$. 


\section{Analytic Families of Semihyperbolic GPLs}

Definition 6.1. Let $\Lambda$ be an open open subset of $\mathbb{C}^{d}$ for some $d \geq 1$. A family $\left\{f_{\lambda}: \mathcal{U}_{\lambda} \rightarrow\right.$ $U\}_{\lambda \in \Lambda}$ of semihyperbolic GPLs is called analytic provided that the following conditions are satisfied.

(a) The index sets $I, I_{o}, I_{c}$ and $I_{r}$ are the same for all $\lambda \in \Lambda$.

(b) For every $i \in I$ the map $\lambda \mapsto \partial U_{\lambda, i} \in \mathcal{D}(\bar{U}), \lambda \in \Lambda$ is continuous, where $\mathcal{D}(\bar{U})$ is the space of all compact subsets of $\bar{U}$ endowed with the Hausdorff metric and $U_{\lambda, i}:=\left(U_{\lambda}\right)_{i}$. Consequently the map $\lambda \mapsto \overline{U_{\lambda, i}}$ is continuous and also the set $(\Lambda * U)_{i}:=\bigcup_{\lambda \in \Lambda}\{\lambda\} \times U_{\lambda, i} \subset \Lambda \times \mathbb{C}$ is open.

(c) Put $\Lambda * U=\bigcup_{i \in I}(\Lambda * U)_{i}$. The map $F: \Lambda * U \rightarrow U$ given by the formula $F(\lambda, z)=$ $f_{\lambda}(z)$ is required to be holomorphic.

(d) For every $i \in I_{c}$ and every $\lambda \in \Lambda$ denote by $c_{\lambda, i}$ the only critical point of the map $f_{\lambda}$ in $U_{\lambda, i}$. The order of critical points $c_{\lambda, i}$ is assumed to be independent of $\lambda$ and is denoted by $q_{i} \geq 2$.

(e) For every $i \in I_{o}$ there exists an open disk $U_{i} \subset U$ containing closures of all sets $U_{\lambda, i}, \lambda \in \Lambda$ and such that

$$
\overline{U_{i}} \cap \overline{\bigcup_{\lambda \in \Lambda} \bigcup_{n \geq 1} f_{\lambda}^{n}\left(\operatorname{Crit}\left(f_{\lambda}\right)\right)}=\emptyset .
$$

As an immediate consequence of items (c), (d) and the Implicit Function Theorem, we get the following.

Lemma 6.2. For every $i \in I_{c}$ the map $\lambda \mapsto c_{\lambda_{i}} \in U, \lambda \in \Lambda$, is holomorphic.

As an immediate consequence of item (b) above and the fact (one of the requirements in the definition of GPL's) that for all $\lambda \in \Lambda, \bigcup_{\in \in I} \overline{U_{\lambda, i}} \subset U$, we get the following.

Lemma 6.3. For every $\gamma \in \Lambda$ there exists $R_{\gamma}>0$ such that

$$
\operatorname{dist}\left(\partial U, \bigcup_{i \in I} \bigcup_{\lambda \in B\left(\gamma, R_{\gamma}\right)} U_{\lambda, i}\right)>0 .
$$

Also, for every compact set $\Gamma \subset \Lambda$, the union $\bigcup_{i \in I} \bigcup_{\lambda \in \Gamma} \overline{U_{\lambda, i}}$ is a compact subset of $U$ and, in particular,

$$
\operatorname{dist}\left(\partial U, \bigcup_{i \in I} \bigcup_{\lambda \in \Gamma} \overline{U_{\lambda, i}}\right)>0 .
$$

Let us state the following obvious lemma.

Lemma 6.4. For every open simply connected set $Q \subset U$, all $\lambda_{0} \in \Lambda$, and all $i \in I_{c}$, such that $f_{\lambda_{0}, i}\left(c_{\lambda_{0}, i}\right) \notin Q$, there exists $r>0$ such that if $f_{\lambda_{0}, i, *}^{-1}: Q \rightarrow \mathbb{C}$ is a holomorphic inverse 
branch of $f_{\lambda_{0}, i}$, then for every $\lambda \in B\left(\lambda_{0}, r\right)$ there exists $f_{\lambda, i, *}^{-1}: Q \rightarrow \mathbb{C}$ a unique holomorphic inverse branch of $f_{\lambda, i}$ such that the $\operatorname{map}(\lambda, z) \mapsto f_{\lambda, i, *}^{-1}(z),(\lambda, z) \in B\left(\lambda_{0}, r\right) \times Q$, is analytic.

We will need a detailed description of derivatives of the inverse branches appearing in the previous lemma. For each $i \in I_{c}$ and every $\lambda \in \Lambda$ there exists a holomorphic map $H_{\lambda, i}: U_{\lambda, i} \rightarrow \mathbb{C}$ such that

$$
f_{\lambda}(z)=\left(z-c_{\lambda, i}\right)^{q_{i}} H_{\lambda, i}(z)+f_{\lambda}\left(c_{\lambda, i}\right) \text { and } H_{\lambda, i}\left(c_{\lambda, i}\right) \neq 0 .
$$

Obviously, the map $(\lambda, z) \mapsto H_{\lambda, i}(z)$ is holomorphic on $(\Lambda * U)_{i}$. Also,

$$
\begin{aligned}
f_{\lambda}^{\prime}(z) & =q_{i}\left(z-c_{\lambda, i}\right)^{q_{i}-1} H_{\lambda, i}(z)+\left(z-c_{\lambda, i}\right)^{q_{i}} H_{\lambda, i}^{\prime}(z) \\
& =\left(z-c_{\lambda, i}\right)^{q_{i}-1}\left(q_{i} H_{\lambda, i}(z)+H_{\lambda, i}^{\prime}(z)\left(z-c_{\lambda, i}\right)\right) .
\end{aligned}
$$

Since $c_{\lambda, i}$ is the only point in $U_{\lambda, i}$ where the derivative $f_{\lambda}^{\prime}$ vanishes, we have

$$
q_{i} H_{\lambda, i}(z)+H_{\lambda, i}^{\prime}(z)\left(z-c_{\lambda, i}\right) \neq 0
$$

for all $z \in U_{\lambda, i}$. Now, let $f_{\lambda, i, *}^{-1}: Q \rightarrow \mathbb{C}$ be a holomorphic inverse branch of $f_{\lambda, i}$ defined on an open simply connected set $Q \subset U \backslash\left\{f_{\lambda}\left(c_{\lambda, i}\right)\right\}$. A straightforward calculation shows that

$$
\left(\left(f_{\lambda, i, *}^{-1}\right)^{\prime}(z)\right)^{q_{i}}=\left(z-f_{\lambda}\left(c_{\lambda, i}\right)\right)^{1-q_{i}} G_{\lambda, i}\left(f_{\lambda, i, *}^{-1}(z)\right)
$$

for all $z \in Q$, where

$$
G_{\lambda, i}(w)=H_{\lambda, i}^{q_{i}-1}(w)\left(q_{i} H_{\lambda, i}(w)+H_{\lambda, i}^{\prime}(w)\left(w-c_{\lambda, i}\right)\right)^{-q_{i}}, w \in U_{\lambda, i} .
$$

Since, by (6.1), $G_{\lambda, i}$ does not vanish throughout $U_{\lambda, i}$ and since the set $U_{\lambda, i}$ is simply connected, there exists $\log _{\lambda} G_{\lambda, i}: U_{\lambda, i} \rightarrow \mathbb{C}$, a holomorphic branch of logarithm of $G_{\lambda, i}$. Clearly, as long as $\Lambda$ is simply connected (we can always assure this by decreasing $\Lambda$ to a round neighborhood of a fixed point), we can choose these branches such that the following holds.

Lemma 6.5. For every $i \in I_{c}$ the function $(\lambda, z) \mapsto \log _{\lambda} G_{\lambda, i}(z),(\lambda, z) \in(\Lambda * U)_{i}$, is holomorphic.

The main result of this section and the ultimate goal of the paper is stated in the following theorem.

Theorem 6.6. If $\Lambda$ is an open open subset of $\mathbb{C}^{d}$ for some $d \geq 1$, and if $\left\{f_{\lambda}: \mathcal{U}_{\lambda} \rightarrow U\right\}_{\lambda \in \Lambda}$ is an analytic family of semihyperbolic GPLs, then we have that the function $\Lambda \ni \lambda \mapsto$ $\mathrm{HD}\left(J\left(f_{\lambda}\right)\right)$ is real-analytic.

Naturally, we want to apply the machinery of weakly regular analytic families of conformal graph directed Markov systems developed in previous sections. Indeed, Let $\Phi^{\lambda}=\Phi_{f_{\lambda}}$, $\lambda \in \Lambda$, be the CGDMS resulting from Proposition 5.3 where the role of the $\tilde{U}_{i}, i \in I_{o}$, appearing in its proof, is played by the disks $U_{i}, i \in I_{o}$, from the Definition 6.1(e). Thus, the seed sets of all the systems $\Phi^{\lambda}, \lambda \in \Lambda$, are the same. Note that because of condition (e) above, each element $\phi_{e}^{\lambda}: U_{\lambda, t(e)} \rightarrow U, e \in E_{\lambda}:=E_{f_{\lambda}}$, extends uniquely to an inverse 
holomorphic branch of $f_{\lambda}^{\|e\|}$ defined on $U_{t(e)}$. This extension will be denoted by the same symbol $\phi_{e}^{\lambda}$. Our goal now is to reparametrize the sets $E_{\lambda}$ so that all the corresponding incidence matrices coincide and all the systems $\Phi^{\lambda}, \lambda \in \Lambda$ form a weakly regular analytic family. Indeed, fix $\gamma \in \Lambda$ and consider a map $\phi_{e}^{\gamma} \in \Phi^{\gamma}, e \in E_{\gamma}$. Suppose first that $\phi_{e}^{\gamma}\left(U_{t(e)}\right) \cap \mathcal{U}_{\gamma, c}=\emptyset$. Then

$$
\phi_{\gamma, e}=f_{\gamma, e_{1}}^{-1} \circ f_{\gamma, e_{2}}^{-1} \circ \ldots f_{\gamma, e_{n}}^{-1}: U_{e_{n+1}}=U_{t(e)} \rightarrow U_{i(e)}=U_{e_{1}}
$$

for some $n \geq 1, e_{2}, e_{3} \ldots, e_{n} \in I_{r}, e_{1} \in I_{r} \cap I_{o}$, and $e_{n+1} \in I_{o}$. Then for every $\lambda \in \Lambda$ the map

$$
f_{\lambda, e_{1}}^{-1} \circ f_{\lambda, e_{2}}^{-1} \circ \ldots f_{\lambda, e_{n}}^{-1}: U_{e_{n+1}}=U_{t(e)} \rightarrow U_{i(e)}=U_{e_{1}}
$$

belongs to $\Phi^{\lambda}$ and there is exactly one vertex $e_{\lambda} \in E_{\lambda}$ such that $f_{\lambda, e_{1}}^{-1} \circ f_{\lambda, e_{2}}^{-1} \circ \ldots f_{\lambda, e_{n}}^{-1}=\phi_{e_{\lambda}}^{\lambda}$. It obviously follows from condition (b) of Definition 6.1 that the map $(\lambda, z) \mapsto \phi_{e_{\lambda}}^{\lambda}(z)$, $(\lambda, z) \in \Lambda \times U_{e_{n+1}}$, is analytic. If

$$
\phi_{e}^{\gamma}\left(U_{t(e)}\right) \cap \mathcal{U}_{\gamma, c} \neq \emptyset, \text { say } \phi_{e}^{\gamma}\left(U_{t(e)}\right) \subset U_{\gamma, k}, k \in I_{c},
$$

then $f_{\gamma} \circ \phi_{e}^{\gamma}=f_{\gamma, e_{1}}^{-1} \circ f_{\gamma, e_{2}}^{-1} \circ \ldots f_{\gamma, e_{n}}^{-1}: U_{e_{n+1}} \rightarrow U_{e_{1}}$ with some $n \geq 0, e_{1}, e_{2}, e_{3} \ldots, e_{n} \in I_{r}$, and $e_{n+1} \in I_{o}$. Taking a sufficiently small ball, say $B(\gamma, R)$, around $\gamma$, we may assume without loss of generality that the set $\Lambda$ is simply connected. We claim that there exists a unique family of maps $\left\{\tilde{\phi}_{\lambda}: U_{e_{n+1}} \rightarrow U_{e_{1}}\right\}_{\lambda \in \Lambda}$ with the following properties holding for all $\lambda \in \Lambda=B(\gamma, R)$.

(f) $f_{\lambda}^{n+1} \circ \tilde{\phi}_{\lambda}=\mathrm{Id}$.

(g) The map $\tilde{\phi}_{(\cdot)}: \Lambda \times U_{e_{n+1}} \rightarrow \mathbb{C}$ is analytic.

(h) $\tilde{\phi}_{\gamma}=\phi_{e}^{\gamma}$.

(i) $f_{\lambda} \circ \tilde{\phi}_{\lambda}=f_{\lambda, e_{1}}^{-1} \circ f_{\lambda, e_{2}}^{-1} \circ \ldots f_{\lambda, e_{n}}^{-1}$.

Put $\psi_{\lambda}=f_{\lambda, e_{1}}^{-1} \circ f_{\lambda, e_{2}}^{-1} \circ \ldots f_{\lambda, e_{n}}^{-1}: U_{e_{n+1}} \rightarrow U_{e_{1}}$ and note that, as above, the map $(\lambda, z) \mapsto$ $\psi_{\lambda}(z)$, is analytic. Let $0 \leq R_{*} \leq R$ be the largest radius such that the family satisfying conditions (f)-(i) is defined for all $\lambda \in B\left(\gamma, R_{*}\right)$. By way of contradiction, suppose that $R_{*}<R$. Consider an arbitrary point $\mu \in \Lambda$ such that $\|\mu-\gamma\|=R_{*}$. Since $f_{\mu}\left(\operatorname{Crit}\left(f_{\mu}\right)\right) \cap$ $\overline{\psi_{\mu}\left(U_{e_{n+1}}\right)}=\emptyset$, all the inverse branches $f_{\mu, k, *}: Q \rightarrow U$ are well-defined on some open simply connected set $Q$ containing $\overline{\psi_{\mu}\left(U_{e_{n+1}}\right)}$. Applying Lemma 6.4 with $\lambda_{0}=\mu$ we get some $r_{\mu}>0$ such that all the branches $f_{\lambda, k, *}^{-1}: Q \rightarrow U$ are well defined for all $\lambda \in B\left(\mu, r_{\mu}\right)$. One can assume $r_{\mu}>0$ to be so small that $\psi_{\lambda}\left(U_{e_{n+1}}\right) \subset Q$ for all $\lambda \in B\left(\mu, r_{\mu}\right)$. Then for every $\lambda \in B\left(\mu, r_{\mu}\right) \cap B\left(\gamma, R_{*}\right)$ there exists $*(\lambda)$, labeling a holomorphic branch of $f_{\mu, k}^{-1}$, such that $\tilde{\phi}_{\lambda}=f_{\lambda, k, *(\lambda)}^{-1} \circ \psi_{\lambda}$. Since the map $F_{*}: B\left(\gamma, R_{*}\right) \times U_{e_{n+1}} \rightarrow U$, given by the formula $F_{*}(\lambda, z)=\tilde{\phi}_{\lambda}(z)$, is holomorphic, the index $*(\lambda)$ is constant on $B\left(\mu, r_{\mu}\right) \cap B\left(\gamma, R_{*}\right)$, say equal to $*$. Thus the formula

$$
F_{\mu}(\lambda, z)= \begin{cases}F_{*}(\lambda, z) & \text { if } \lambda \in B\left(\gamma, R_{*}\right) \\ f_{\lambda, k, *}^{-1} \circ \psi_{\lambda}(z) & \text { if } \lambda \in B\left(\mu, r_{\mu}\right)\end{cases}
$$

defines a holomorphic function on $B\left(\gamma, R_{*}\right) \cup B\left(\mu, r_{\mu}\right)$. If now $\mu_{1}$ and $\mu_{2}$ are two arbitrary points in $\Lambda$ such that $\left\|\mu_{2}-\gamma\right\|=\left\|\mu_{1}-\gamma\right\|=R_{*}$ and $B\left(\mu_{1}, r_{\mu_{1}}\right) \cap B\left(\mu_{2}, r_{\mu_{2}}\right) \neq \emptyset$, then 


$$
\begin{aligned}
& B\left(\mu_{1}, r_{\mu_{1}}\right) \cap B\left(\mu_{2}, r_{\mu_{2}}\right) \cap B\left(\gamma, R_{*}\right) \neq \emptyset \text { and } \\
& \left.\quad F_{\mu_{2}}\right|_{\left(B\left(\mu_{1}, r_{\mu_{1}}\right) \cap B\left(\mu_{2}, r_{\mu_{2}}\right) \cap B\left(\gamma, R_{*}\right)\right) \times U_{e_{n+1}}}=\left.F_{\mu_{1}}\right|_{\left(B\left(\mu_{1}, r_{\mu_{1}}\right) \cap B\left(\mu_{2}, r_{\mu_{2}}\right) \cap B\left(\gamma, R_{*}\right)\right) \times U_{e_{n+1}} .} .
\end{aligned}
$$

Since $F_{\mu_{1}}$ and $F_{\mu_{2}}$ are holomorphic, we therefore conclude that

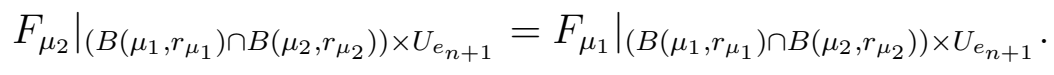

Thus the formula

$$
F(\lambda, z)= \begin{cases}F_{*}(\lambda, z) & \text { if } \lambda \in B\left(\gamma, R_{*}\right) \\ F_{\mu}(\lambda, z) & \text { if }\|\mu-\gamma\|=R_{*} \text { and } \lambda \in B\left(\mu, r_{\mu}\right)\end{cases}
$$

defines a holomorphic function on the open connected set $Z=B\left(\gamma, R_{*}\right) \cup \bigcup_{\|\mu-\gamma\|=R_{*}} B\left(\mu, r_{\mu}\right)$. Obviously, the family $\left\{\tilde{\phi}_{\lambda}:=F(\lambda, \cdot)\right\}_{\lambda \in Z}$ satisfies the conditions (f)-(i). Since the sphere $\left\{\mu \in \Lambda:\|\mu-\gamma\|=R_{*}\right\}$ is compact, there exists $R^{\prime} \in\left(R_{*}, R\right]$ such that $B\left(\gamma, R^{\prime}\right) \subset Z$. This contradiction finishes the proof of the equality $R_{*}=R$.

Thus, each map $\tilde{\phi}^{\lambda}$ is a member of $\Phi^{\lambda}$, i.e. there exists a unique element $e_{\lambda} \in E_{\lambda}$ such that $\tilde{\phi}^{\lambda}=\phi_{e_{\lambda}}^{\lambda}$ and the map $(\lambda, z) \mapsto \phi_{e_{\lambda}}^{\lambda}(z)$ is analytic. Summarizing the two cases above, we have thus defined a function $e \mapsto e_{\lambda}$ from $E_{\gamma}$ to $E_{\lambda}$ with the following properties.

(j) $e_{\gamma}=e$.

(k) The map $e \mapsto e_{\lambda}$ from $E_{\gamma}$ to $E_{\lambda}$ is bijective.

(l) The map $(\lambda, z) \mapsto \phi_{e_{\lambda}}^{\lambda}(z)$ from $U_{e_{n+1}}$ to $\mathbb{C}$ is analytic.

We now set $E=E_{\gamma}$ and identify the elements of $E_{\lambda}$ with elements of $E$ via the bijective map $e \mapsto e_{\lambda}$. We have thus proved the following.

Lemma 6.7. The family $\left\{\Phi^{\lambda}\right\}_{\lambda \in \Lambda}$ is analytic.

Our aim now is to show that the family $\left\{\Phi^{\lambda}\right\}_{\lambda \in \Lambda}$ is weakly regular analytic. For every $\omega=\omega_{1} \omega_{2} \ldots \omega_{n} \in I_{r}^{*}$ and every $\lambda \in \Lambda$, put

$$
f_{\lambda, \omega}^{-1}=f_{\lambda, \omega_{1}}^{-1} \circ f_{\lambda, \omega_{2}}^{-1} \circ \ldots f_{\lambda, \omega_{n}}^{-1}: U \rightarrow U_{\lambda, \omega_{1}} .
$$

We start with the following.

Lemma 6.8. For every compact set $\Gamma \subset \Lambda$ and every compact set $V \subset U$ there exist $\alpha>0$ and $C_{1}>0$ such that

$$
\left|\left(f_{\lambda, \omega}^{-1}\right)^{\prime}(z)\right| \leq C_{1} e^{-\alpha\|\omega\|}
$$

for all $\lambda \in \Gamma$, all $\omega \in I_{r}^{*}$ and all $z \in V$.

Proof. Because of condition (b) of Definition 6.1, the union

$$
W=\bigcup_{i \in I_{r}} \bigcup_{\lambda \in \Gamma} \overline{U_{\lambda, i}}
$$

is a compact subset of $U$. There thus exists $\kappa \in(0,1)$ such that all the maps $f_{l, i}^{-1}: V \rightarrow$ $U_{l, i} \subset W \subset U, i \in I_{r}$, decrease hyperbolic distances on $V$ and $W$ (viewed as compact subsets of the hyperbolic surface $U$ ) by the constant factor $\kappa$. It follows from this and 
compactness of $V$ and $W$ that there exist $\beta<1$ and $q \geq 1$ such that $\left|\left(f_{\lambda, \tau}^{-1}\right)^{\prime}(z)\right| \leq \beta$ for all $(\lambda, \tau, z) \in \Gamma \times I_{r}^{q} \times W$. Hence, noting that $\sup \left\{\left|\left(f_{\lambda, i}^{-1}\right)^{\prime}(z)\right|:(\lambda, i, z) \in \Gamma \times I_{r} \times W\right\}<+\infty$, our lemma follows.

Shrinking all the sets $U_{i}, i \in I_{o}$, slightly, all the requirements imposed on them in Definition 6.1 will be satisfied, and all these sets will be compactly contained in the original sets $U_{i}$. Then, in the same way as Lemma 6.8, we prove the following.

Lemma 6.9. For every compact set $\Gamma \subset \Lambda$ there exist $\beta>0$ and $C_{2}>0$ such that

$$
\left|\left(\phi_{\omega}^{\lambda}\right)^{\prime}(z)\right| \leq C_{2} e^{-\beta|\omega|}
$$

for all $\lambda \in \Gamma$, all $\omega \in E_{A}^{*}$ and all $z \in U_{t(\omega)}$.

We end this section with the following two results.

Lemma 6.10. If $\Gamma$ is a compact subset of $\Lambda$, then for every $i \in I_{c}$ the closure of the set $\Gamma_{i}^{*}:=\bigcup_{\lambda \in \Gamma} \bigcup_{\substack{\omega \in E_{A}^{*} \\ \omega_{1,1}=i}}\{\lambda\} \times \phi_{\omega}^{\lambda}\left(U_{\lambda, t(\omega)}\right)$ is a compact subset of $\bigcup_{\lambda \in \Lambda}\{\lambda\} \times U_{\lambda, i}$.

Proof. Consider an arbitrary sequence $\left(\lambda_{n}, x_{n}\right)_{n=1}^{\infty} \in \Gamma_{i}^{*}$. Since $\Gamma$ is compact, we can assume without loss of generality that the sequence $\left(\lambda_{n}\right)_{n=1}^{\infty}$ converges in $\Gamma$, say to $\lambda_{\infty}$. Since $\bar{U}$ is compact, we can assume further that the sequence $\left(x_{n}\right)_{n=1}^{\infty}$ converges, say to some point $x_{\infty} \in \bar{U}$. Since $x_{n} \in U_{\lambda_{n}, i}$ for all $n \geq 1$, it follows from condition (b) of Definition 6.1 that $x_{\infty} \in \overline{U_{\lambda_{\infty}, i}}$. We want to show that $x_{\infty} \in U_{\lambda_{\infty}, i}$. Since the set $I_{o}$ is finite, we may assume without loss of generality that $f_{\lambda_{n}}\left(x_{n}\right) \in U_{\lambda_{n}, k}$ for some $k \in I_{o}$ and all $n \geq 1$. Suppose now for the contrary that $x_{\infty} \in \partial U_{\lambda_{\infty}, i}$. Then $F\left(\lambda_{\infty}, x_{\infty}\right) \in \partial U$ and therefore,

$$
\lim _{n \rightarrow \infty} \operatorname{dist}\left(f_{\lambda_{n}}\left(x_{n}\right), \partial U\right)=\lim _{n \rightarrow \infty} \operatorname{dist}\left(F\left(\lambda_{n}, x_{n}\right), \partial U\right)=\operatorname{dist}\left(F\left(\lambda_{\infty}, x_{\infty}\right), \partial U\right)=0
$$

contrary to the fact that $f_{\lambda_{n}}\left(x_{n}\right) \in U_{\lambda_{n}, k}$ and $\operatorname{dist}\left(\bigcup_{j \in I_{o}} \bigcup_{\lambda \in \Gamma} U_{\lambda_{j}}, \partial U\right)>0$ (see Lemma 6.3). We are done.

As an immediate consequence of Lemma 6.10 and Lemma 6.5, we get the following.

Lemma 6.11. For every compact subset $\Gamma$ of $\Lambda$ the number

$$
M_{\Gamma}=\max _{i \in I_{c}}\left\{\sup \left\{\left|\log _{\lambda} G_{\lambda, i}(z)\right|: z \in \Gamma_{i}^{*}\right\}\right\}
$$

is finite. 


\section{The $\psi$ FUnCTION}

We shall show in this section that the condition (d) in the definition of weakly regular analyticity is satisfied for the family $\left\{\Phi^{\lambda}\right\}_{\lambda \in \Lambda}$ of CGDMS defined in the previous section. As a direct consequence of the last assertion of Lemma 4.1, we get the following.

Lemma 7.1. The family $\left\{\lambda \mapsto f_{\lambda, \tau}^{-1}\left(\pi_{\lambda}(\omega)\right): \omega \in E_{A}^{\infty}, \tau \in I_{r}^{*}\right\}$ consists of holomorphic maps and is normal (since bounded).

Fix $\gamma \in \Lambda$ (called $\lambda_{0}$ in Section 4). Recall that we have defined the function $\psi$, for each $\omega \in E_{A}^{\infty}$, by the formula

$$
\psi_{\omega}(\lambda)=\frac{\left(\phi_{\omega_{1}}^{\lambda}\right)^{\prime}\left(\pi_{\lambda}(\sigma \omega)\right)}{\left(\phi_{\omega_{1}}^{\gamma}\right)^{\prime}\left(\pi_{\gamma}(\sigma \omega)\right)} .
$$

The goal of this section is to prove the following.

Lemma 7.2. There exists $R_{*}>0$ such that for every $\omega \in E_{A}^{\infty}$ there exists $\log \psi_{\omega}$ : $B\left(\gamma, R_{*}\right) \rightarrow \mathbb{C}$, a holomorphic branch of logarithm of $\psi_{\omega}$, such that $\log \psi_{\omega}(\gamma)=0$ and such that the family of functions $\left\{\lambda \mapsto \frac{1}{\left\|\omega_{1}\right\|} \log \psi_{\omega}(\lambda)\right\}_{\omega \in E_{A}^{\infty}}$ is bounded and, consequently, normal.

Proof. Since there are only finitely many elements $e \in E$ with $\|e\|=1$, we can assume without loss of generality that $\left\|\omega_{1}\right\| \geq 2$. Take $R_{1}>0$ so small that $\bar{B}\left(\gamma, R_{1}\right) \subset \Lambda$. Then $\bar{B}\left(\gamma, R_{1}\right)$ is compact and, by Lemma 6.3 , the set

$$
W=\bigcup_{i \in I} \bigcup_{\lambda \in \bar{B}\left(\gamma, R_{1}\right)} \overline{U_{\lambda, i}} \subset U
$$

is compact. Thus, for every $i \in I_{r}$ the function $(\lambda, z) \mapsto\left(f_{\lambda, i}^{-1}\right)^{\prime}(z)$ restricted to the Cartesian product $\bar{B}\left(\gamma, R_{1}\right) \times W$ is uniformly continuous. Therefore, since $I_{r}$ is a finite set and since these functions are nowhere vanishing, there exists $R_{2} \in\left(0, R_{1}\right]$ such that

$$
\left|\frac{\left(f_{\lambda, i}^{-1}\right)^{\prime}(z)}{\left(f_{\gamma, i}^{-1}\right)^{\prime}(\xi)}-1\right|<\frac{1}{4}
$$

for all $i \in I_{r}$, all $\lambda \in B\left(\gamma, R_{2}\right)$ and all $(z, \xi) \in W$ with $|z-\xi| \leq R_{2}$. In view of Lemma 7.1, there exists $R_{3} \in\left(0, R_{2}\right]$ such that

$$
\left|f_{\lambda, \tau}^{-1}\left(\pi_{\lambda}(\omega)\right)-f_{\gamma, \tau}^{-1}\left(\pi_{\gamma}(\omega)\right)\right|<R_{2}
$$

for all $\omega \in E_{A}^{\infty}$, all $\tau \in I_{r}^{*}$, and all $\lambda \in B\left(\gamma, R_{3}\right)$. Since

$$
\bigcup_{\lambda \in \bar{B}\left(\gamma, R_{3}\right)} \pi_{\lambda}\left(E_{A}^{\infty}\right) \subset \bigcup_{\lambda \in \bar{B}\left(\gamma, R_{1}\right)} \pi_{\lambda}\left(E_{A}^{\infty}\right) \subset W,
$$


and since $f_{\lambda, \tau}^{-1}(W) \subset W$ for all $\tau \in I_{r}^{*}$, and all $\lambda \in B\left(\gamma, R_{3}\right)$, combining (7.1) and (7.2), we conclude that

$$
\left|\frac{\left(f_{\lambda, i}^{-1}\right)^{\prime}\left(f_{\lambda, \tau}^{-1}\left(\pi_{\lambda}(\omega)\right)\right)}{\left(f_{\gamma, i}^{-1}\right)^{\prime}\left(f_{\gamma, \tau}^{-1}\left(\pi_{\lambda}(\omega)\right)\right)}-1\right|<\frac{1}{4}
$$

for all $i \in I_{r}$, all $\omega \in E_{A}^{\infty}$, all $\tau \in I_{r}^{*}$, and all $\lambda \in B\left(\gamma, R_{3}\right)$. In particular, there exists

$$
\log \left(\frac{\left(f_{\lambda, i}^{-1}\right)^{\prime}\left(f_{\lambda, \tau}^{-1}\left(\pi_{\lambda}(\omega)\right)\right)}{\left(f_{\gamma, i}^{-1}\right)^{\prime}\left(f_{\gamma, \tau}^{-1}\left(\pi_{\lambda}(\omega)\right)\right)}\right)
$$

a holomorphic branch of logarithm of the function

$$
\lambda \mapsto \frac{\left(f_{\lambda, i}^{-1}\right)^{\prime}\left(f_{\lambda, \tau}^{-1}\left(\pi_{\lambda}(\omega)\right)\right)}{\left(f_{\gamma, i}^{-1}\right)^{\prime}\left(f_{\gamma, \tau}^{-1}\left(\pi_{\lambda}(\omega)\right)\right)}, \lambda \in B\left(\gamma, R_{3}\right),
$$

whose value at $\gamma$ is equal to 0 . Note that $M_{1}$, the supremum of moduli of these logarithms, is finite. Now, if $\omega \in E_{A}^{\infty}$ and $\omega_{1,1} \notin I_{c}$, then for all $\lambda \in B\left(\gamma, R_{3}\right)$, we set

$$
\log \psi_{\omega}(\lambda)=\sum_{j=1}^{\left\|\omega_{1}\right\|} \log \left(\frac{\left(f_{\lambda, \omega_{1, j}}^{-1}\right)^{\prime}\left(f_{\lambda, \bar{\omega}_{j}}\left(\pi_{\lambda}(\sigma(\omega))\right)\right)}{\left(f_{\gamma, \omega_{1, j}}^{-1}\right)^{\prime}\left(f_{\gamma, \bar{\omega}_{j}}\left(\pi_{\gamma}(\sigma(\omega))\right)\right)}\right),
$$

where $\bar{\omega}_{j}=\omega_{1, j+1} \omega_{1, j+2} \ldots \omega_{1,\left\|\omega_{1}\right\|} \in I_{r}^{\left\|\omega_{1}\right\|-j}$ for all $1 \leq j \leq\left\|\omega_{1}\right\|-1$ and $\bar{\omega}_{1,\left\|\omega_{1}\right\|}=\emptyset$. So,

$$
\left|\log \psi_{\omega}(\lambda)\right| \leq M_{1}|| \omega_{1}||
$$

If $\omega_{1,1} \in I_{c}$, then write $f_{\lambda}\left(c_{\lambda, \omega_{1,1}}\right)=v_{\lambda}$ and put

$$
\log _{\omega}^{(0)}(\lambda)=\sum_{j=2}^{\left\|\omega_{1}\right\|} \log \left(\frac{\left(f_{\lambda, \omega_{1, j}}^{-1}\right)^{\prime}\left(f_{\lambda, \bar{\omega}_{j}}\left(\pi_{\lambda}(\sigma(\omega))\right)\right)}{\left(f_{\gamma, \omega_{1, j}}^{-1}\right)^{\prime}\left(f_{\gamma, \bar{\omega}_{j}}\left(\pi_{\gamma}(\sigma(\omega))\right)\right)}\right)
$$

and we have

$$
\left|\log _{\omega}^{(0)}(\lambda)\right| \leq M_{1}\left(|| \omega_{1} \|-1\right) .
$$

Write $f \circ \varphi_{\omega_{1}}^{\lambda}=\left.f_{\lambda, \tau}^{-1}\right|_{U_{k}}$, where $\tau=\omega_{2} \omega_{3} \ldots \omega_{|\omega|} \in I_{r}^{\left|\omega_{1}\right| \mid-1}$ and $k=t\left(\omega_{1}\right) \in I_{o}$. Put $n=\|\tau\|=\left|\omega_{1}\right| \mid-1$. Then $f_{\lambda}^{n}\left(v_{\lambda}\right) \notin U_{k}$. Since $W$ is a compact subset of $U$ and in virtue of item (d) of Definition 6.1, we have

$$
\Delta_{1}:=\min \left\{\operatorname{dist}(W, \partial U), \operatorname{dist}\left(\bigcup_{i \in I_{o}} \overline{U_{i}}, \bigcup_{\lambda \in \Lambda} \bigcup_{j \geq 1} f_{\lambda}^{j}\left(\operatorname{Crit}\left(f_{\lambda}\right)\right)\right)\right\}>0 .
$$

Hence, for $j \geq 0$ and for all $\lambda \in \Lambda$,

$$
\operatorname{dist}\left(U_{k}, \partial U\right), \operatorname{dist}\left(U_{k}, f_{\lambda}^{j}\left(v_{\lambda}\right)\right) \geq \Delta_{1}>0 .
$$

Since all the mappings $f_{\lambda, j}^{-1}, j \in I_{r}$, are conformal homeomorphisms, the moduli of their derivatives restricted to $W$ are uniformly bounded away from zero and infinity. Hence, using (7.4) and Lemma 6.8, we conclude that there exist a universal integer $p \geq 1$ and a constant $\Delta_{2}>0$, such that

$$
B\left(f_{\lambda,\left.\tau\right|_{n-p+1} ^{n}}^{-1}\left(U_{k}\right), \Delta_{2}\right) \subset U
$$


and

$$
\begin{aligned}
\Delta_{1} & \leq \operatorname{dist}\left(f_{\lambda,\left.\tau\right|_{n-p+1} ^{n}}^{-1}\left(U_{k}\right), f_{\lambda}^{n-p}\left(v_{\lambda}\right)\right) \leq \operatorname{diam}\left(f_{\lambda,\left.\tau\right|_{n-p+1} ^{n}}^{-1}\left(U_{k}\right) \cup\left\{f_{\lambda}^{n-p}\left(v_{\lambda}\right)\right\}\right) \\
& \leq K_{3}^{-1} \min \{\kappa, \pi / 24\} \Delta_{2},
\end{aligned}
$$

where $\kappa>0$ is so small such that

$$
\max \left\{\frac{1+\kappa}{(1-\kappa)^{3}},\left(\frac{1-\kappa}{(1+\kappa)^{3}}\right)^{-1}\right\} \leq \sqrt{2}
$$

and $K_{3}$ comes from Theorem 4.1.2 in [7]. Note that $f_{\lambda}^{n-p}\left(v_{\lambda}\right)=f_{\lambda,\left.\tau\right|_{n-p+1} ^{n}}^{-1}\left(f_{\lambda}^{n-p}\left(v_{\lambda}\right)\right)$. Put

$$
g_{\lambda}:=f_{\lambda,\left.\tau\right|_{n-p}}^{-1} \text { and } \rho_{\lambda}:=f_{\lambda,\left.\tau\right|_{n-p+1} ^{n}}^{-1} .
$$

Using (7.5) and (7.6) and applying Theorem 4.1.5 from [7], we get that

$$
\begin{aligned}
& \left|\frac{g_{\lambda}\left(\rho_{\lambda}\left(\pi_{\lambda}(\sigma(\omega))\right)\right)-v_{\lambda}}{g_{\lambda}^{\prime}\left(\rho_{\lambda}\left(\pi_{\lambda}(\sigma(\omega))\right)\right)\left(f_{\lambda}^{n-p}\left(v_{\lambda}\right)-\rho_{\lambda}\left(\pi_{\lambda}(\sigma(\omega))\right)\right.}-1\right|= \\
& \quad=\left|\frac{g_{\lambda}\left(\rho_{\lambda}\left(\pi_{\lambda}(\sigma(\omega))\right)\right)-g_{\lambda}\left(f_{\lambda}^{n-p}\left(v_{\lambda}\right)\right)}{g_{\lambda}^{\prime}\left(\rho_{\lambda}\left(\pi_{\lambda}(\sigma(\omega))\right)\left(f_{\lambda}^{n-p}\left(v_{\lambda}\right)-\rho_{\lambda}\left(\pi_{\lambda}(\sigma(\omega))\right)\right)\right.}-1\right| \leq \\
& \quad \leq K_{3} \Delta_{2}^{-1}\left|f_{\lambda}^{n-p}\left(v_{\lambda}\right)-\rho_{\lambda}\left(\pi_{\lambda}(\sigma(\omega))\right)\right| \\
& \quad \leq \min \{\kappa, \pi / 24\}<1 / 3 .
\end{aligned}
$$

Let $\log _{0}: B(1,1) \rightarrow \mathbb{C}$ be the holomorphic branch of the logarithmic function determined by the requirement that $\log _{0} 1=0$. By $(7.7)$, the following composition $\log _{\omega}^{(1)}: B\left(\gamma, R_{3}\right) \rightarrow \mathbb{C}$, given by the formula

$$
\log _{\omega}^{(1)}(\lambda)=\log _{0}\left(\frac{g_{\lambda}\left(\rho_{\lambda}\left(\pi_{\lambda}(\sigma(\omega))\right)\right)-v_{\lambda}}{g_{\lambda}^{\prime}\left(\rho_{\lambda}\left(\pi_{\lambda}(\sigma(\omega))\right)\right)\left(f_{\lambda}^{n-p}\left(v_{\lambda}\right)-\rho_{\lambda}\left(\pi_{\lambda}(\sigma(\omega))\right)\right)}\right),
$$

is well define. Furthermore, it follows from (7.7) that

$$
\left\|\log _{\omega}^{(1)}\right\|_{\infty} \leq M_{2}:=\sup \left\{\left|\log _{0}(z)\right|: z \in \bar{B}(1,1 / 3)\right\} .
$$

The same considerations as those leading us to (7.3) provide us with a holomorphic branch of the logarithm $\log _{\omega}^{(2)}: B\left(\gamma, R_{3}\right) \rightarrow \mathbb{C}$ of the function $\lambda \mapsto g_{\lambda}^{\prime}\left(\rho_{\lambda}\left(\pi_{\lambda}(\sigma(\omega))\right)\right) / g_{\gamma}^{\prime}\left(\rho_{\gamma}\left(\pi_{\gamma}(\sigma(\omega))\right)\right)$, $\lambda \in B\left(\gamma, R_{3}\right)$, such that

$$
\left\|\log _{\omega}^{(2)}\right\|_{\infty} \leq M_{1}(n-p) \leq M_{1}\left\|\omega_{1}\right\| .
$$

it follows from (7.6) and Koebe's Distortion Theorem (the sets $U_{k}$ are uniformly compactly contained in $U)$ that there exist $R_{4} \in\left(0, R_{3}\right]$ sufficiently small, a constant $M_{3}>0$ and $\log _{\omega}^{(3)}: B\left(\gamma, R_{4}\right) \rightarrow \mathbb{C}$, a holomorphic branch of the logarithm of the function $\lambda \mapsto$ $f_{\lambda}^{n-p}\left(v_{\lambda}\right)-\rho_{\lambda}\left(\pi_{\lambda}(\sigma(\omega))\right)$, such that

$$
\left\|\log _{\omega}^{(3)}\right\|_{\infty} \leq M_{3} .
$$


Since

$$
\begin{aligned}
& \frac{g_{\lambda}\left(\rho_{\lambda}\left(\pi_{\lambda}(\sigma(\omega))\right)\right)-v_{\lambda}}{g_{\gamma}\left(\rho_{\gamma}\left(\pi_{\gamma}(\sigma(\omega))\right)\right)-v_{\lambda}}= \\
& \quad=\frac{g_{\lambda}\left(\rho_{\lambda}\left(\pi_{\lambda}(\sigma(\omega))\right)\right)-v_{\lambda}}{g_{\lambda}^{\prime}\left(\rho_{\lambda}\left(\pi_{\lambda}(\sigma(\omega))\right)\right)\left(f_{\lambda}^{n-p}\left(v_{\lambda}\right)-\rho_{\lambda}\left(\pi_{\lambda}(\sigma(\omega))\right)\right)} \cdot \frac{g_{\lambda}^{\prime}\left(\rho_{\lambda}\left(\pi_{\lambda}(\sigma(\omega))\right)\right)}{g_{\gamma}^{\prime}\left(\rho_{\gamma}\left(\pi_{\gamma}(\sigma(\omega))\right)\right)} \cdot \\
& \quad \frac{f_{\lambda}^{n-p}\left(v_{\lambda}\right)-\rho_{\lambda}\left(\pi_{\lambda}(\sigma(\omega))\right)}{f_{\gamma}^{n-p}\left(v_{\lambda}\right)-\rho_{\gamma}\left(\pi_{\gamma}(\sigma(\omega))\right)} \cdot \frac{g_{\gamma}^{\prime}\left(\rho_{\gamma}\left(\pi_{\gamma}(\sigma(\omega))\right)\left(f_{\gamma}^{n-p}\left(v_{\lambda}\right)-\rho_{\gamma}\left(\pi_{\gamma}(\sigma(\omega))\right)\right)\right.}{g_{\gamma}\left(\rho_{\gamma}\left(\pi_{\gamma}(\sigma(\omega))\right)\right)-v_{\lambda}}
\end{aligned}
$$

the function $\log _{\omega}^{(4)}(\lambda)=\log _{\omega}^{(1)}(\lambda)+\log _{\omega}^{(2)}(\lambda)+\log _{\omega}^{(3)}(\lambda)-\log _{\omega}^{(3)}(\gamma)-\log _{\omega}^{(1)}(\gamma)$ is a holomorphic branch of the logarithm of the function $\lambda \mapsto\left(g_{\lambda}\left(\rho_{\lambda}\left(\pi_{\lambda}(\sigma(\omega))\right)\right)-v_{\lambda}\right) /\left(g_{\gamma}\left(\rho_{\gamma}\left(\pi_{\gamma}(\sigma(\omega))\right)\right)-\right.$ $\left.v_{\lambda}\right), \lambda \in B\left(\gamma, R_{4}\right)$, and it follows from (7.8)-(7.10) that

$$
\left\|\log _{\omega}^{(4)}\right\|_{\infty} \leq 2 M_{2}+M_{1}\left\|\omega_{1}\right\|+2 M_{3}
$$

Since $\log _{\omega}^{(4)}(\gamma)=2 \pi i l$ with some integer $l$, we thus get that $|2 \pi i l| \leq M_{1}|| \omega_{1}||+2\left(M_{2}+M_{3}\right)$. Therefore, using (7.11) again, we conclude that $\log _{\omega}^{(5)}(\lambda):=\log _{\omega}^{(4)}(\lambda)-2 \pi i l$ is a holomorphic branch of logarithm of the function $\lambda \mapsto\left(g_{\lambda}\left(\rho_{\lambda}\left(\pi_{\lambda}(\sigma(\omega))\right)\right)-v_{\lambda}\right) /\left(g_{\gamma}\left(\rho_{\gamma}\left(\pi_{\gamma}(\sigma(\omega))\right)\right)-v_{\lambda}\right)$ such that

$$
\log _{\omega}^{(5)}(\gamma)=0 \text { and }\left\|\log _{\omega}^{(5)}\right\|_{\infty} \leq 2\left(M_{1}\left\|\omega_{1}\right\|+2\left(M_{2}+M_{3}\right)\right)
$$

Let now $f_{\lambda, *}^{-1}: f_{\lambda, \tau}^{-1}\left(U_{k}\right) \rightarrow U_{\lambda, \omega_{1,1}}$ be the holomorphic inverse branch of $f$ determined by the requirement that $\left.f_{\lambda, *}^{-1} \circ f_{\lambda, \tau}^{-1}\right|_{U_{k}}=\phi_{\omega_{1}}^{\lambda}$. Put $G_{\lambda}=G_{\lambda, \omega_{1,1}}$ and $q=q_{\omega_{1,1}}$. It follows from (6.2), Lemma 6.10, Lemma 6.11, and (7.12) that the function $\log _{\omega}^{(6)}: B\left(\gamma, R_{4}\right) \rightarrow \mathbb{C}$, given by the formula

$$
\lambda \mapsto \frac{1-q}{q} \log _{\omega}^{(5)}(\lambda)+\frac{1}{q}\left(\log G_{\lambda}\left(\pi_{\lambda}(\sigma(\omega))\right)-\log G_{\lambda}\left(\pi_{\gamma}(\sigma(\omega))\right)\right),
$$

is a holomorphic branch of logarithm of the function

$$
\lambda \mapsto \frac{f_{\lambda, *}^{-1}\left(g_{\lambda}\left(\rho_{\lambda}\left(\pi_{\lambda}(\sigma(\omega))\right)\right)\right)}{f_{\gamma, *}^{-1}\left(g_{\gamma}\left(\rho_{\gamma}\left(\pi_{\gamma}(\sigma(\omega))\right)\right)\right.}
$$

and

$$
\left\|\log _{\omega}^{(6)}\right\|_{\infty} \leq 2\left(1-\frac{1}{q_{c}}\right)\left(M_{1}\left\|\omega_{1}\right\|+2\left(M_{2}+M_{3}\right)\right)+2 M_{4},
$$

where $M_{4}=M_{\bar{B}\left(\gamma, R_{4}\right)}$ is the number $M_{\Gamma}$ obtained in Lemma 6.11. Combining this with (7.3), we see that $\log \psi_{\omega}=\log _{\omega}^{(0)}+\log _{\omega}^{(6)}$ and

$$
\left\|\log _{\omega}^{(6)}\right\|_{\infty} \leq 2\left(\left(2-\frac{1}{q_{+}}\right) M_{1}\left\|\omega_{1}\right\|+\left(1-\frac{1}{q_{+}}\right)\left(M_{2}+M_{3}\right)+M_{4}\right),
$$

where $q_{+}=\max \left\{q_{i}: i \in I_{c}\right\}$. We are done. 


\section{Conclusion of the Proof of the Main Theorem}

In this section we complete the proof of Theorem 6.6. Keeping the notation from the proof of Lemma 7.2 we begin with by showing that condition (c) of weak regular analyticity (see the beginning of Section 4) is satisfied for the family $\mathcal{F}=\left\{\Phi^{\lambda}\right\}_{\lambda \in B\left(\gamma, R_{*}\right)}$ from Section 6 with $\kappa(e)=-\beta\|e\|$ with some constant $\beta>0$. Putting $q_{-}=\min \left\{q_{i}: i \in I_{c}\right\}$, note that it follows from $(6.2),(7.7),(7.6)$ and Lemma 6.10 that

$$
\begin{aligned}
\mid f_{\lambda, c}^{-1}\left(g_{\lambda}\right. & \left.\left(\rho_{\lambda}\left(\pi_{\lambda}(\sigma(\omega))\right)\right)\right) \mid \leq \\
& \leq\left\|G\left|\|_{\infty}^{1 / q}\right| g_{\lambda}\left(\rho_{\lambda}\left(\pi_{\lambda}(\sigma(\omega))\right)\right)-\left.v_{\lambda}\right|^{\frac{1}{q}-1}\right. \\
& \leq(3 / 2)^{1-\frac{1}{q}}\|G\|_{\infty}^{1 / q}\left|g_{\lambda}^{\prime}\left(\rho_{\lambda}\left(\pi_{\lambda}(\sigma(\omega))\right)\right)\right|^{\frac{1}{q}-1}\left|f_{\lambda}^{n-p}\left(v_{\lambda}\right)-\rho_{\lambda}\left(\pi_{\lambda}(\sigma(\omega))\right)\right|^{\frac{1}{q}-1} \\
& \leq(3 / 2)^{1-\frac{1}{q}}\|G\|_{\infty}^{1 / q} \Delta_{1}^{\frac{1}{q}-1}\left|g_{\lambda}^{\prime}\left(\rho_{\lambda}\left(\pi_{\lambda}(\sigma(\omega))\right)\right)\right|^{\frac{1}{q}-1},
\end{aligned}
$$

where $\left.\|G\|_{\infty}=\max \left\{1, \max _{i \in I_{c}} \sup \left\{\| G_{\lambda}(z) \mid:(\lambda, z) \in(\bar{B}(\gamma, R) * U)_{i}\right\}\right\}\right\}$. Therefore,

$$
\begin{aligned}
\left|\phi_{\omega_{1}}^{\lambda}\left(\pi_{\lambda}(\sigma(\omega))\right)\right| & =\left|f_{\lambda, *}^{-1}\left(g_{\lambda}\left(\rho_{\lambda}\left(\pi_{\lambda}(\sigma(\omega))\right)\right)\right)\right|\left|g_{\lambda}^{\prime}\left(\rho_{\lambda}\left(\pi_{\lambda}(\sigma(\omega))\right)\right)\right| \cdot\left|\rho_{\lambda}^{\prime}\left(\pi_{\lambda}(\sigma(\omega))\right)\right| \\
& \leq\left(2 D_{1} / 3\right)^{\frac{1}{q}-1}\left|g_{\lambda}^{\prime}\left(\rho_{\lambda}\left(\pi_{\lambda}(\sigma(\omega))\right)\right)\right|^{\frac{1}{q}} \cdot\left|\rho_{\lambda}^{\prime}\left(\pi_{\lambda}(\sigma(\omega))\right)\right| \\
& \leq M_{6}\left|g_{\lambda}^{\prime}\left(\rho_{\lambda}\left(\pi_{\lambda}(\sigma(\omega))\right)\right)\right|^{\frac{1}{q}}
\end{aligned}
$$

where $M_{6}=\left(2 D_{1} / 3\right)^{\frac{1}{q_{-}}-1} \max ^{p}\left\{\left\|\left(f_{j}^{-1}\right)^{\prime}\right\|_{\infty}: j \in I_{r}\right\}$. Finally, applying Lemma 6.8, we get

$$
\begin{aligned}
\left|\phi_{\omega_{1}}^{\lambda}\left(\pi_{\lambda}(\sigma(\omega))\right)\right| & \leq\|G\|_{\infty}^{\frac{1}{q}} M_{6} \exp \left(-\frac{\alpha}{q}\left(\left\|\omega_{1}\right\|-(p+1)\right)\right) \\
& \leq\|G\|_{\infty}^{\frac{1}{q_{-}}} M_{6} \exp \left(-\frac{\alpha}{q_{-}}\left(\left\|\omega_{1}\right\|-(p+1)\right)\right) \\
& \leq C_{2} e^{-\beta\left\|\omega_{1}\right\|}
\end{aligned}
$$

for all $\omega \in E_{A}^{\infty}$ (also those for which $\left\|\omega_{1}\right\|=1$ ), all $\lambda \in B\left(\gamma, R_{4}\right)$ and some universal constants $C_{2} \geq 1$ and $\beta>0$.

Item (b) of weakly regular analyticity of the family $\mathcal{F}$, i.e. strong regularity of the system $\Phi^{\gamma}$, follows easily from Lemma 4.5 in [13]. Indeed, we know that $\mathrm{P}\left(\operatorname{HD}\left(J_{\Phi^{\gamma}}\right)=0\right.$, and, by Proposition 5.3), $u:=\operatorname{HD}\left(J_{\Phi^{\gamma}}\right)=\operatorname{HD}\left(J\left(f_{\gamma}\right)\right)$. So, applying Lemma 4.5 in [13] for the pair $(s, u)$, where $s=0$, we obtain the strong regularity of the system $\Phi^{\gamma}$. Now, combining this fact along with Lemma 7.2 (condition (d) of weakly regular analyticity), (8.1) (condition (c)) and Lemma 6.7, we conclude that the familiy $\mathcal{F}=\left\{\Phi^{\lambda}\right\}_{\lambda \in B\left(\gamma, R_{*}\right)}$ is weakly regular analytic. Therefore, Theorem 6.6 follows now immediately from Theorem 4.2 and Proposition 5.3.

\section{EXAMPLES}

In this section we give two classes of analytic families of semihyperbolic generalized polynomiallike mappings. In the first one the critical point and the critical disk vary, whereas in the second one, the regular maps vary. The first example is inspired by a construction in [6]. 
Example 1. Suppose that $g: \mathcal{U}_{r} \rightarrow U=B(0,1)$ is a GPL without branching (critical) points, consisting of affine maps. Fix an integer $q \geq 2$, a point $v \in J(g)$ and $\xi \in U \backslash \overline{\mathcal{U}_{r}}$. Take $R>0$ so small that $\bar{B}\left(\xi, 2 R \subset U \backslash \overline{\mathcal{U}_{r}}\right.$. Let $H: B(0,1) \rightarrow B(0,1)$ be the conformal homeomorphism of the unit disk $B(0,1)$ onto itself given by the formula $H(z)=\frac{z+v}{1+\bar{v} z}$. Define the maps $f_{\lambda}: \mathcal{U}_{r} \cup B\left(\xi+\lambda_{1}, \lambda_{2}\right) \rightarrow B(0,1), \lambda=\left(\lambda_{1}, \lambda_{2}\right) \in B(0, R) \times(B(0, R) \backslash\{0\})$, by the formula

$$
f_{\lambda}(z)= \begin{cases}g(z) & \text { if } z \in \mathcal{U}_{r} \\ H\left(\frac{\left(z-\left(\xi+\lambda_{1}\right)\right)^{q}}{\lambda_{2}^{q}}\right) & \text { if } z \in B\left(\xi+\lambda_{1}, \lambda_{2}\right) .\end{cases}
$$

Obviously, all the maps $f_{\lambda}$ are generalized polynomial-like mappings. Furthermore, $\xi+\lambda_{1}$ is the only critical point of $f_{\lambda}$ and $f_{\lambda}\left(\xi+\lambda_{1}\right)=v$. Since $v \in J(g)$, since $f_{\lambda}(J(g))=$ $J(g)$, since $B\left(\xi+\lambda_{1}, \lambda_{2}\right) \subset B(\xi, 2 R)$ and since $J(g) \cap B(\xi, 2 R)=\emptyset$, all the maps $f_{\lambda}$ are semihyperbolic and all conditions (a)-(e) of Definition 6.1 are satisfied. This means that $\left\{f_{\lambda}\right\}_{\lambda \in B(0, R) \times(B(0, R) \backslash\{0\})}$ is an analytic family of semihyperbolic GPLs and, as a consequence of Theorem 6.6, we get that the map $\lambda \mapsto \operatorname{HD}\left(J\left(f_{\lambda}\right)\right), \lambda \in B(0, R) \times(B(0, R) \backslash\{0\})$, is real-analytic.

Example 2. Consider a hyperbolic GPL $f: \mathcal{U}_{r} \rightarrow U$ with the following two properties.

(a) $f(f(c))=f(c)$ for every critical point $c$ of $f$.

(b) If $c_{1}, c_{2}$ are two different critical points of $f$ and $f\left(c_{1}\right) \in U_{i_{1}}, f\left(c_{2}\right) \in U_{i_{2}}$, then $i_{1} \neq i_{2}$.

For every critical point $c$ of $f$ let $i_{c} \in I$ be the only element of $I$ such that $f(c) \in I_{i(c)}$. Put $\hat{I}=\{j(c): c \in \operatorname{Crit}(f)\}$. For every $c \in \operatorname{Crit}(f)$, let $R_{j(c)}: B(0,1) \rightarrow U_{j(c)}$ be a conformal homeomorphism sending 0 to $f(c)$. For every $\lambda \in B(0,1)$, consider the map $f_{\lambda}: \mathcal{U}_{r} \rightarrow \bigcup_{j \in \hat{I}} R_{j}(B(0,1))$ given by the formula

$$
f_{\lambda}(z)= \begin{cases}f(z) & \text { if } z \notin \bigcup_{j \in \hat{I}} U_{j} \\ f\left(R_{j}\left(\lambda^{-1} R_{j}^{-1}(z)\right)\right) & \text { if } z \in R_{j}(B(0, \lambda)) \text { and } j \in \hat{I} .\end{cases}
$$

Since for every $\lambda \in B(0,1), \operatorname{Crit}\left(f_{\lambda}\right)=\operatorname{Crit}(f)$ and $f_{\lambda}\left(f_{\lambda}(c)\right)=f(c)=f_{\lambda}(c)$ for every $c \in \operatorname{Crit}(f)$, it is straightforward to verify that $\left\{f_{\lambda}\right\}_{\lambda \in B(0,1)}$ is an analytic family of semihyperbolic GPLs and, as a consequence of Theorem 6.6, we get that the map $\lambda \mapsto \operatorname{HD}\left(J\left(f_{\lambda}\right)\right)$, $\lambda \in B(0,1)$, is real-analytic.

\section{REFERENCES}

[1] E. Hille, Analytic function theory, II. AMS Chelsea Publishing (2002), 2nd Edition.

$[2]$ T. Kato, Perturbation theory for linear operators, Springer (1995).

[3] J. Kotus, M. Urbański, Fractal Measures and Ergodic Theory of Transcendental Meromorphic Functions, Preprint 2004, to appear London Math. Soc. Lect. Notes.

[4] N. Makarov, S. Smirnov, Phase transition in subhyperbolic Julia sets, Ergod. Th. \& Dynam. Sys. 16 (1996), 125-157.

[5] N. Makarov, S. Smirnov, On "thermodynamics" of rational maps, I. Negative spectrum, Comm. Math. Phys. 211 (2000), 705-743.

[6] N. Makarov, S. Smirnov, On "thermodynamics" of rational maps, II. Non-recurrent maps. Journal of London Math. Soc. 67 (2003), 417-432. 
[7] R. D. Mauldin, M. Urbański, Graph directed Markov systems: geometry and dynamics of limit sets, Cambridge Univ. Press (2003).

[8] V. Mayer, M. Urbański, Geometric Thermodynamical Formalism and Real Analyticity for Meromorphic Functions of Finite Order, Ergod. Th. \& Dynam. Sys. 28 (2008), 915 - 946.

[9] V. Mayer, M. Urbański, Thermodynamical Formalism and Multifractal Analysis for Meromorphic Functions of finite order, Preprint 2006, to appear Memoirs of AMS.

[10] M. Roy, M. Urbański, Regularity properties of Hausdorff dimension in conformal infinite IFS, Ergod. Th. \& Dynam. Sys. 25 (2005), 1961-1983.

[11] W. Rudin, Functional Analysis, McGraw-Hill, Inc. (1991) 2nd Edition.

[12] D. Ruelle, Repellers for real analytic maps. Ergod. Th. \& Dynam. Sys. 2 (1982), 99-107.

[13] B. Stratmann, M. Urbański, Real analyticity of topological pressure for parabolically semihyperbolic generalized polynomial-like maps, Indag. Mathem. 14 (2003), 119-134.

[14] B. Stratmann, M. Urbański, Multifractal analysis for parabolically semihyperbolic generalized polynomial-like maps, IP New Studies in Advanced Mathematics 5 (2004), 393-447.

[15] M. Urbański, A. Zdunik, Real analyticity of Hausdorff dimension of finer Julia sets of exponential family, Ergod. Th. \& Dynam. Sys.24 (2004), 279-315.

[16] M. Urbański, M. Zinsmeister, Geometry of hyperbolic Julia-Lavaurs sets, Indagationes Math. 12 (2001) $273-292$.

Mariusz Urbański, Department of Mathematics, University of North Texas, Denton, TX 76203-1430, USA

E-mail address: urbanskiunt.edu

Web: www.math.unt.edu/ urbanski 